Published in final edited form as:

Nat Cell Biol. 2021 May 01; 23(5): 485-496. doi:10.1038/s41556-021-00676-z.

\title{
Dynamic adult tracheal plasticity drives stem cell adaptation to changes in intestinal homeostasis in Drosophila
}

\author{
Jessica Perochon ${ }^{1}$, Yachuan $\mathrm{Yu}^{1,2}$, Gabriel N. Aughey ${ }^{3}$, André B. Medina ${ }^{1,2}$, Tony D. \\ Southall ${ }^{3}$, Julia B. Cordero ${ }^{1,2,{ }^{*}}$ \\ ${ }^{1}$ Institute of Cancer Sciences-University of Glasgow, Wolfson Wohl Cancer Research Centre \\ Garscube Estate, Switchback Road, Glasgow, G61 1QH \\ ${ }^{2}$ Cancer Research UK Beatson Institute, Garscube Estate, Switchback Road, Glasgow, G61 1BD \\ ${ }^{3}$ Department of Life Sciences, Imperial College London, London, SW7 2AZ
}

\section{Abstract}

Coordination of stem cell function by local and niche-derived signals is essential to preserve adult tissue homeostasis and organismal health. The vasculature is a prominent component of multiple stem cell niches. However, its role in adult intestinal homeostasis remains largely understudied. Here, we uncover a previously unrecognised crosstalk between adult intestinal stem cells (ISCs) in Drosophila and the vasculature-like tracheal system, which is essential for intestinal regeneration. Following damage to the intestinal epithelium, gut-derived reactive oxygen species (ROS) activate tracheal HIF-1 $a$ and bidirectional FGF/FGFR signalling, leading to reversible remodelling of gutassociated terminal tracheal cells and ISC proliferation following damage. Unexpectedly, ROSinduced adult tracheal plasticity involves downregulation of the tracheal specification factor trachealess (trh) and upregulation of IGF2 mRNA-binding protein (IGF2BP2/Imp). Our results reveal an intestine/vasculature inter-organ communication programme, which is essential to adapt stem cells response to the proliferative demands of the intestinal epithelium.

Adult intestinal plasticity depends on the action of stem cells, which respond to signals from the intestinal epithelium and its microenvironment, to fulfil global tissue demands ${ }^{1-3}$. Little is known about the role of the vascular microenvironment in intestinal homeostasis.

Users may view, print, copy, and download text and data-mine the content in such documents, for the purposes of academic research, subject always to the full Conditions of use: http://www.nature.com/authors/editorial_policies/license.html\#terms

*Corresponding author: Julia.Cordero@glasgow.ac.uk.

Reporting Summary

Further information on research design, including blinding and randomization is available in the Nature Research Reporting Summary linked to this Article.

Author contributions.

J.P designed and carried out most experiments and analysed and interpreted the data. Y.Y. provided technical support throughout the study and perform RT-qPCRs. J.P. G.A, T.S and J.B.C. analysed the TaDa data. A.B.M. performed the experiments in Extended Data Fig.5k-p. J.B.C conceived the project, designed experiments, analysed the data and supervised the study. J.P and J.B.C wrote the paper with contributions from the rest of the authors.

Competing Interests

The authors declare no competing interests. 
The Drosophila tracheal system is an oxygen-delivering interconnected tubular network, analogous to the mammalian vascular and respiratory systems ${ }^{4}$. Following specification from epidermal cells and the formation of a tracheal sac in the embryo, tracheal cells undergo extensive cell rearrangements and cell shape changes, leading to the formation of multicellular tubes that ramify into progressively thinner branches, culminating with a terminal tracheal cell (TTCs) ${ }^{5}$. Drosophila TTCs, analogous to mammalian vascular tip cells $^{6}$, extend prominent cytoplasmic projections, which supply oxygen to their target tissues ${ }^{4,5,7,8}$. While tracheal development and post-embryonic plasticity have been significantly studied $^{5,9,10}$, there is scarce knowledge on the role and regulation of the adult tracheal system.

The adult Drosophila melanogaster midgut shares remarkable homology with the mammalian intestine ${ }^{11}$. Critically, the midgut epithelium is maintained by intestinal stem cells (ISCs), which self-renew and replenish the differentiated intestinal lineage—secretory enteroendocrine cells and absorptive enterocytes-through the production of undifferentiated enteroblasts ${ }^{12,13}$. The Drosophila gastrointestinal tract is densely tracheated ${ }^{14}$. Beyond the requirement for tracheal derived Dpp/BMP to restrain ISC proliferation ${ }^{15}$, there is no knowledge on the role of the tracheal system in adult midgut biology.

Here, we combine genetics and image analysis with in vivo functional and molecular studies to characterise an inter-organ communication programme between the adult Drosophila midgut and its closely associated tracheal tissue, which is essential to shape stem cell and tracheal plasticity during intestinal regeneration.

\section{Results}

\section{Intestinal damage induces reversible gut TTC remodelling}

The adult Drosophila midgut is covered by TTCs, labelled with a $G A L 4$ reporter driven by the Serum Response Factor (dSRF) ${ }^{16-18}(d S R F>G F P$ ) (Fig.1a and Extended data Fig.1a). Transmission electron microscopy denoted intimate contact between TTCs, enterocytes (ECs) (Fig.1b) and ISCs (Fig.1c). Oxygen and nutrients influence TTC plasticity ${ }^{10,19}$. We noticed that damage to the adult Drosophila midgut epithelium, caused by feeding animals with the pathogenic bacteria Pseudomonas entomophila $(P e)^{20-23}$, the DNA-damaging agent Bleomycin ${ }^{24-26}$, or the epithelial basement membrane disruptor Dextran Sulfate Sodium $(\mathrm{DSS})^{25,27}$, led to a significant increase in TTC coverage within the posterior midgut (Fig.1d, e and Extended data Fig.1b-e). Single TTC clones confirmed the increase in total number of cellular branches derived from individual TTCs in damaged $(P e)$ versus control (Sucrose) midguts (Fig.1f, g). Interestingly, TTC clones within $P e$ treated midguts revealed direct correlation between the number of individual TTC branches and nearby $\mathrm{PH}^{+}$ISCs (Fig.1h). Further quantification of tracheal phenotypes showed increase in primary, secondary and tertiary tracheal branches and total length of individual TTC extensions in damaged $(P e)$ versus control (Sucrose) midguts (Fig.1i-k). We observed no evidence of TTC proliferation following midgut damage (Extended data Fig.1f, g). Collectively, these data suggest extensive cellular remodelling of TTCs in response to epithelial intestinal injury. A time course assessment of posterior midguts over a 16 hour period of $P e$ infection (Damage 
phase) followed by $32 \mathrm{hrs}$ on normal diet (Recovery phase) revealed direct correlation between tracheal coverage and ISC proliferation (Fig.11, m and Extended data Fig.1h, i). These results strongly suggest that adult gut-associated-tracheal remodelling is a highly dynamic and reversible process, which accompanies changes in intestinal homeostasis.

Low doses of whole body $\gamma$-irradiation in mice induce intestinal epithelial cell death, followed by a strong peak of crypt cell proliferation between 72- and $96 \mathrm{hrs}$ after irradiation $^{28}$. Staining with anti-CD31, showed an increase in vascular endothelial cells in regenerating (irradiated) versus control (non-irradiated) intestinal crypts (Fig.1n). These results indicate a conserved phenomenology of vasculature/tracheal response to damage in the adult intestinal epithelium.

\section{TTC remodelling drives ISC proliferation upon gut damage}

An essential step in the intestinal regenerative response to damage involves a robust increase in ISC proliferation 1, 2022, 25, 29 (Fig.1d, h). To address the functional role of the tracheal system in adult intestinal regeneration, we severely reduced TTC numbers by overexpressing the pro-apoptotic gene bax (UAS-bax) using temperature sensitive $d S R F-G a l 4$ ( $d S R F^{t s}>$ bax $)$ (Fig.2a, b). This impaired midgut regeneration, as evidenced by approximately $50 \%$ decrease in ISC proliferation following (Fig.2c). TTCs are best known for their role in facilitating gas exchange with their target tissues ${ }^{4}$. Thus, poor intestinal regeneration following TTC reduction might reflect the need for oxygen in this process. Hypoxic environmental conditions induced activity of a reporter of Drosophila Hypoxia-inducible factor-1a (HIF-1a/Sima) ${ }^{30}$ and TTC remodelling in the adult midgut (Extended data Fig.2ac). However, hypoxia did not induce ISC proliferation in the absence of gut damage (Fig.2d). While hypoxia impaired damage induced ISC proliferation in the adult midgut (Fig.2d), it did so to a lower extent than that observed upon TTC loss (dSRFts $>$ bax $)$ (Fig.2c). This difference could be due to a compensatory effect of increased trachea upon hypoxia (Extended data Fig.2a, c) or compromised ISC survival in $d S R F^{t s}>$ bax midguts. We assessed apoptosis and ISC numbers in hypoxic and $d S R F^{t s}>$ bax midguts through anti-caspase (Dcp-1) staining and the use of an ISC reporter (Delta-LacZ). Midguts overexpressing bax in adult ECs ( $N P 1^{t s}>$ bax $)$, served as a 'cell-death' positive control (Extended data Fig.2d, f). While we saw no evidence of cell death in hypoxic midguts, $d S R F^{t s}>b a x$ midguts showed significant apoptosis (Extended data Fig.2e, g), which was restricted to ECs, distinguished by their large nuclei (Extended data Fig.2e, lower panel, magnified view). Consistently, this cell death phenotype did not translate into defective ISC numbers (Extended data Fig.2h, i). Therefore, impaired midgut regeneration following the hypoxia or TTC ablation regime used in our study is unlikely to be secondary to ISC loss. Alternatively, differences in the regenerative response of hypoxic versus $d S R F^{t s}>$ bax midguts could be explained by the contribution of angiocrine factors to ISC proliferation, in addition to oxygen availability. Dpp/BMP ligand has been identified as an angiocrine factor in the adult midgut ${ }^{31}$. However, its action inhibits rather than induces ISC proliferation ${ }^{31}$. Therefore, a potential role of Dpp cannot directly explain our results.

Tracheal remodelling and ISC proliferation show almost identical dynamics (Fig.11, $\mathrm{m}$ and Extended data Fig. 1h, i). To address whether these events are part of a feedforward 
mechanism or if one precedes the other, we assessed tracheal remodelling following damage while blocking ISC proliferation by overexpressing $U A S$-myc RNAi $\left(m y c^{-I R}\right)^{32}$ using the stem/progenitor driver escargot-GAL4 ( esg $^{t s}>m y c^{-I R}$ ) (Fig.2e-g). As we needed to use the $G A L 4 / U A S$ system to genetically manipulate gut cells, we established a scoring method for assessing tracheal coverage through the use of light microscopy, which was validated against our confocal microscopy tracheal quantification approach (Extended Data Fig.3a-c). Gutassociated trachea remodelled normally in $e^{t s} g^{t s}>m y c^{-I R}$ midguts following $P e$ damage, in spite of the almost complete absence of ISC proliferation (Fig.2e-g). Therefore, TTC remodelling precedes midgut ISCs proliferation following damage. We hypothesised that signals activated by damage upstream of ISC proliferation might induce gut-tracheal remodelling.

\section{Gut-derived ROS induces TTC remodelling through HIF-1a/FGFR}

Pathogen-induced intestinal damage triggers a strong oxidative burst and the production of reactive oxygen species (ROS) from the intestinal epithelium ${ }^{33}, 34$. We tested whether ROS could trigger tracheal remodelling in the regenerating intestine. Systemic impairment of ROS by feeding animals with the antioxidant N-acetyl cysteine (NAC) or genetically blocking ROS production in ECs by overexpressing the enzyme catalase ${ }^{33}$ ( $N P 1^{t s}>$ catalase) inhibited damage-induced tracheal remodelling (Fig.2h, i and Extended data Fig.3d, e) and regenerative ISC proliferation (Fig. $2 \mathrm{j}$ and Extended data Fig. $3 \mathrm{f})^{33}$. Conversely, driving adult intestinal epithelial cell death through bax overexpression in ECs $\left(N P 1^{t s}>b a x\right)$ was sufficient to induce TTC remodelling and ISC proliferation (Extended data Fig.3g-i). Therefore, intestinal epithelial damage and ROS induce remodelling of gut associated trachea, which is in turn necessary to drive ISC proliferation during intestinal regeneration.

Exogenous $\mathrm{H}_{2} \mathrm{O}_{2}$ can stabilize HIF-1a - a key conserved driver of hypoxia-induced tracheal/vascular remodelling $9,35,36$ - in normoxia ${ }^{37}$. The Sima/HIF-1a activity reporter $l d h-l a c Z$ was upregulated in gut-associated TTCs following midgut damage and in a ROS dependent manner (Fig.2k-m). Furthermore, midguts from sima ${ }^{-/}$whole mutant animals or upon adult specific sima knockdown within TTCs $\left(d S R F^{t s}>s i m a^{-I R}\right)$ showed impaired tracheal remodelling and ISC proliferation following damage (Fig.3a-f).

The Drosophila fibroblast growth factor receptor (FGFR), Breathless (Btl), is a well-known transcriptional target of HIF-1a during tracheal development and oxygen-driven tracheal remodelling ${ }^{19,38}$. Consistently, a reporter of breathless (btl) expression (btl-lacZ) showed gene upregulation in TTCs following intestinal damage, which was abrogated by NAC (Fig.3g-i). TTC knockdown of $b t I\left(d S R F^{t s}>b t t^{I R}\right)$ inhibited tracheal remodelling and ISC proliferation following damage (Fig.3j-1), without evidence of cell death or ISC loss (Extended data Fig.2e, g-i). Therefore, ROS-dependent activation of HIF-1a/FGFR signaling within TTCs following gut damage induces tracheal remodelling and regenerative ISC proliferation in the adult midgut. Consistently, expression of the HIF-1a/FGFR target gene blistered $(b s) / d S R F^{19,39}$ was upregulated in damaged and hypoxic midguts (Extended data Fig.4a-e) and knocking down $b s$ in adult TTCs $\left(d S R F^{t s}>b s^{-I R}\right)$ impaired tracheal remodelling and ISC proliferation following damage (Extended data Fig.4f-h). 


\section{FGF/FGFR drives ISC proliferation and TTC remodelling}

During development or hypoxia, the Drosophila FGF-like ligand Branchless (Bnl) is upregulated in target tissues and signals paracrinally to its receptor FGFR/Breathless (Btl) in the trachea to induce their remodelling $16,19,40$. Consistently, we observed upregulation of a $b n l$ reporter ( $b n l-l a c Z$ ) in ISCs/EBs and ECs following intestinal damage, which was impaired by NAC (Fig.4a-c). Therefore, ROS induces Bnl activation within the intestinal epithelium following damage. Unexpectedly, we also observed $b n l$ upregulated in TTCs following intestinal damage, in an ROS dependent manner (Fig.4d-e). Expression of $b n l$ in TTCs was confirmed by the use of an independent reporter (Extended data Fig.5a, b). Overexpressing $b n l$ in adult TTCs $\left(d S R F^{t s}>b n I\right)$ induced ISC proliferation without TTC remodelling (Extended data Fig.5c, d).

We next assessed the functional role of individual sources of FGF/Bnl in our system. Consistent with our reporter expression data (Fig.4a-e), knocking down bnl from either TTCs $\left(d S R F^{t s}>b n I^{-I R}\right)$, ISCs/EBs $\left(e s g^{t s}>b n I^{I R}\right)$ or ECs $\left(N P I^{t s}>b n I^{I R}\right)$ restrained ISC proliferation following midgut damage but did not impair TTC remodelling (Fig.4f-h and Extended data Fig.5e-j). This is in line with the high sensitivity of the regenerative intestine to discrete fluctuations in individual signaling activity ${ }^{32,41}$. Hence, small variations in $\mathrm{Bnl}$ levels, which are insufficient to affect tracheal remodelling are enough to impact ISC proliferation following damage. Instead, concomitant $b n l$ knockdown from ECs and ISCs/EBs (NP1>, es $g^{t s}>b n I^{I R}$ ) impaired TTC remodelling and ISC proliferation (Fig.4i-k). Therefore, combined action of gut-derived sources of Bnl is necessary to induce tracheal remodelling following intestinal damage. Overexpressing $b n l$ in adult ISCs/EBs ( $e s g^{t s}>b n l$ ) or ECs $\left(N P 1^{t s}>b n l\right)$ was sufficient to induce ISC proliferation and TTC remodelling (Extended data Fig.5k-p).

Given that multiple sources of Bnl—from the midgut and TTCs—can individually contribute to regenerative ISC proliferation independently of tracheal remodelling, we hypothesised this may be through a non-tracheal receptor. Consistently, knocking down btl from ISCs/EBs (esg $\left.g^{t s}>b t l-I R\right)$ prevented ISC proliferation upon damage without affecting TTC remodelling (Extended data Fig.6a-c). In the context of tracheal development, Bnl/Btl signals through the MAPK/ERK pathway, ${ }^{52}$, which is a key driver of ISC proliferation in the adult midgut ${ }^{43-45}$. Knocking down $b t l$ from ISCs/EBs ( $e s g^{t s}>b t I^{-I R}$ ) impaired damageinduced MAPK/ERK activation in the midgut (Extended data Fig.6d, e), suggesting that activation of $\mathrm{Btl}$ in the midgut regulates regenerative ISC proliferation through MAPK/ERK signaling.

\section{Identification of TTC changes triggered upon gut damage}

We next used Targeted DamID (TaDa) for TTC in vivo profiling of RNA Pol II chromatin binding ${ }^{46}$ in control (Sucrose) and Pe treated midguts (Fig.5a and Extended data Fig.7a). $\mathrm{TaDa}$ is particularly advantageous in our system due to inherent difficulties to efficiently separate tracheal tissue from the midgut. We identified 1747 and 1712 genes significantly bound by RNA Pol II in TTCs from control (Sucrose) and Pe infected midguts, respectively (Supplementary Table 1). Gene ontology (GO) analysis of areas with significant RNA Pol II binding in control midguts revealed enrichment in components of the tracheal system, and 
genes previously involved in epithelial tube morphogenesis and respiratory/tracheal system development (Fig.5b) (Supplementary Tables 2 and 3). This validated the sensitivity of TaDa to reliably detect tracheal specific genes from combined gut and tracheal tissue samples.

Consistent with our reporter expression and functional data (Fig.4d-h), TaDa analysis identified $b n l / F G F$ as a gene with significant RNA Pol II binding in Pe treated midguts only (Supplementary Table 1) (Extended Data Fig.7b). Unexpectedly, we were unable to detect significant RNA Pol II binding to $b t l$ in adult TTCs of Pe treated midguts (Supplementary Table 1). This is counterintuitive given our gene expression and functional data on $b t$ (Fig.3g-1). Discrepancies between RNA pol II occupancy and mRNA levels are possible and could be due to pausing of the polymerase ${ }^{47}$, post-transcriptional mRNA regulation ${ }^{48}$ or temporally dynamic RNA pol II binding (e.g during intestinal damage), which may not be captured by a single time point assessment.

\section{Imp/IGF2BP regulates TTC remodelling and midgut regeneration}

We noticed that, within the genes showing significant RNA pol II binding in TTCs of $\mathrm{Pe}$ treated midguts only (Supplementary Table 1), there were several genes associated with neuronal function (Fig.5c). Amongst them, was the highly conserved mRNA-binding protein Imp/IGF2BP (Fig.5d) (Supplementary Table 1), which regulates axonal remodelling in Drosophila ${ }^{49}, 50$. We confirmed upregulation of Imp transcription by RT-qPCR (Fig.5e) and Imp increase in TTCs through the use of a protein trap (Imp::GFP) (Fig.5f, h). NAC treatment showed that Imp upregulation in TTCs following intestinal damage depends on ROS production (Fig.5g, h). Importantly, adult specific knock down of Imp from TTCs $\left(d S R F^{t s}>I m p^{-I R}\right)$ significantly impaired tracheal remodelling and ISC proliferation following midgut damage (Fig.5i-k). There are two known post-transcriptional targets of Imp: chickadee/profilin ${ }^{49}$ and $m y c^{50}$. We found that Myc was upregulated in TTCs of Pe treated midguts and this was abrogated by $\operatorname{Imp}$ knockdown $\left(d S R F^{t s}>i m p^{-I R}\right.$ ) (Fig.6a, b). Adult specific knockdown of myc within TTCs using RNAi ${ }^{32}\left(d S R F^{t s}>m y c^{-I R}\right)$, impaired tracheal remodelling and ISC proliferation following gut damage (Fig.6c-e). Altogether, these results establish Imp as a regulator of TTC remodelling and ISC proliferation during adult Drosophila midgut regeneration. This function of Imp is at least in part through tracheal intrinsic control of Myc.

\section{Trh downregulation is necessary for adult TTC remodelling}

While our TaDa analysis revealed significant binding of RNA Pol II to trachealess (trh) in TTCs of Sucrose treated midguts (Supplementary Table 1 and 3), this was not the case in the damaged tissues (Fig.7a and Supplementary Table 1). This was surprising given that $t r h$ is known a master regulator of tracheal gene expression and it is present in all tracheal cells from the onset of embryonic development through adulthood ${ }^{51-53}$. Loss of trh during development impairs tracheal cell specification and tube morphogenesis ${ }^{52}$, 53. However, RTqPCR (Fig.7b), antibody staining (Fig.7c, d) and a transgenic reporter (trh-lacZ) (Fig.7e, f) confirmed downregulation of thr expression and reduced protein in TTCs upon gut damage. Remarkably, trh-lacZ signal was restored upon $P e$ and NAC co-treatment or $32 \mathrm{hrs}$ after removal of the damaging agent (Fig.7e, f). This suggests that trh expression in adult TTCs is highly dynamic and its downregulation upon intestinal damage is dependent on ROS. 
Importantly, consistent with our gene and protein expression data, trh overexpression in adult TTCs $\left(d S R F^{t s}>t r h\right)$ significantly impaired tracheal remodelling and ISC proliferation (Fig.7g-i), while trh knockdown ( $d S R F^{t s}>t r h^{-I R}$ ) potentiates TTC remodelling and ISC proliferation following midgut damage (Fig.7g-i). Altogether, these results suggest that ROS-induced trh downregulation in adult TTCs is necessary to allow gut associated TTC plasticity and robust regeneration of the intestine following damage.

Here, we reveal an inter-organ communication programme in Drosophila, involving the adult tracheal system and the midgut, which drives reciprocal adaptation of both tissues to sustain a robust regenerative response of the intestine to injury (Fig.7j). Our results may reflect vasculature/stem cell interactions in the mammalian intestine and other self-renewing tissues.

\section{Discussion}

The vasculature represents a prominent component of the gut microenvironment. However, its functional role in adult intestinal homeostasis remains largely unknown. Here, we report the cellular and molecular underpinnings of an inter-organ communication programme between the adult Drosophila midgut and its closely associated vasculature-like tracheal tissue, which is fundamental to drive the regenerative response of ISCs following epithelial tissue damage.

ROS are key initiators of TTC remodelling and ISC proliferation, following intestinal damage by pathogenic infection (Fig. 7j). While our observations show a conserved phenomenology of TTCs/vasculature changes upon diverse intestinal insults and across species, it is highly conceivable that damage and species-specific molecular responses exist and that signals other than or in addition to ROS influence tracheal/vascular adaptations to intestinal damage. Furthermore, blocking ROS systemically by oral administration of NAC, was more potent at inhibiting TTC remodelling than genetically impairing intestinal ROS production (Fig.2h, i and Extended data Fig.3d, e); suggesting a potential cell autonomous effect of the antioxidant within TTCs and role of TTC-derived ROS in the system. In fact, a related study published in this issue, reports intestinal and tracheal intrinsic activation of ROS and HIF-1a as necessary events driving ISC proliferation and tracheal remodelling during intestinal regeneration and tumourigenesis in Drosophila ${ }^{54}$.

HIF-1a/FGFR signaling induces tracheogenesis during development and in response to hypoxia, following activation by FGF from target tissues $9,35,36$. As such, our findings suggest a repurposing of this developmental pathway during adult gut/tracheal crosstalk. Furthermore, we discover ROS-inducible angiocrine Bnl/FGF activating stem/progenitor cell FGFR signaling during adult intestinal regeneration (Fig. 7j). These findings were recapitulated by colleagues in the accompanying study ${ }^{54}$. FGF induces vascular endothelial cell differentiation in human intestinal organoids ${ }^{55}$ and acts as an angiocrine factor in various tumour settings ${ }^{56}$. This highlights the great degree of conservation between the Drosophila and mammalian system and raises the possibility of a conserved angiocrine role of FGF ligands in mammalian intestinal regeneration. 
In addition to the well-established role of oxygen, nutrition regulates TTC remodelling in the larval and adult Drosophila midgut ${ }^{10}$. In this context, a defined subset of enteric neurons influence TTC remodelling through the delivery of Insulin- and Vasoactive Intestinal Peptide-like neuropeptides ${ }^{10}$. Nutrient induced-tracheal remodelling involves activation of Insulin Receptor (InR) signaling within TTCs. Interestingly, FGF/FGFR signaling does not appear to mediate nutrient dependent TTC remodelling ${ }^{10}$; suggesting that molecular events driving tracheal tissue plasticity are diverse and highly dependent on the biological context and/or stimuli.

We identified two tracheal-intrinsic molecular mechanisms triggered in response to intestinal epithelial damage and necessary to induce TTC remodelling and ISC proliferation (Fig. 7j). One, involving upregulation of Drosophila IGF2 mRNA-binding protein (Imp) and its downstream target Myc (Fig. 7j). The other, requiring downregulation of the tracheal cell specification factor trh (Fig. 7j). Known functions of Imp had been restricted to the induction of neuronal remodelling and growth ${ }^{49}, 50$. Its mammalian orthologue, IGF2BP2, has been studied for its involvement in metabolic disease ${ }^{57}$ and its potential role in the vasculature remains to be addressed. Trh, homologous to mammalian NPAS3 ${ }^{58}$, has been exclusively known for its requirement in the specification of tracheal cells from undifferentiated progenitors in the developing embryo ${ }^{51-53}$. Here, we report a role of Trh in terminally differentiated adult tracheal cells, which involves its unexpected downregulation. Emerging evidence suggests that adult tissues and cells, such as the intestine and neurons, lose differentiation markers and acquire 'naïve' or 'foetal-like properties' during the process of tissue regeneration ${ }^{59-61}$. Our work suggests the exciting possibility that this may also be the case for the adult vasculature.

The vasculature is a largely uncharacterized component of the adult intestinal niche. Vascularization of in vitro organ culture systems has been notoriously difficult, representing a major roadblock in the field of tissue engineering. As such, our in vivo findings may be of broad interest and impact to the vascular and intestinal research fields.

\section{Methods}

\section{Fly stocks and rearing}

A complete list of fly lines and full genotypes used in this study can be found in Supplementary Tables 5 and 6. In experiments using the $G A L 4 / G A L 80^{t s}$ system, flies were crossed, $\mathrm{F} 1$ progenies reared, and adults aged for 5 days after eclosion at $18^{\circ} \mathrm{C}$. Animals were then transferred to $29^{\circ} \mathrm{C}$ for 5-7 days to allow activation of most transgenes prior to phenotypic analysis. The exception was bax, which was overexpressed for only 3 days. If not carrying temperature sensitive transgenes, crosses and offspring were kept at $25^{\circ} \mathrm{C}$. Overall, experimental animals were used 10-12 days following adult eclosion. Animals for experiments were maintained in food vials at low densities (10-15 flies per vial) and were transferred to fresh food every 2 days. Only adult posterior midguts from mated females were analysed in this study. 


\section{Damage induced intestinal regeneration}

10-day-old females of the desired genotypes were starved in empty vials for $2 \mathrm{hrs}$ followed by feeding with a 5\% sucrose solution only (Sucrose), or Sucrose containing either $\mathrm{Pe}$ at OD 100, 25 $\mu \mathrm{g} / \mathrm{ml}$ Bleomycin (Sigma-Aldrich, Cat\#B2434), or 3\% DSS (Sigma-Aldrich, Cat\#42867) applied on filter paper discs (Whatman). Pe infection was carried out for 16hrs, Bleomycin feeding was done for 1-day and DSS feeding lasted for 2-days, with fresh media applied each day.

\section{NAC treatment}

Flies were placed in empty vials with a filter paper soaked with a $5 \%$ sucrose solution containing 20mM NAC (Sigma-Aldrich, Cat\#A7250) for $24 \mathrm{hrs}$. Animals were then fed with either $5 \%$ sucrose + NAC or $5 \%$ sucrose + NAC $+P e(O D 100)$ for an additional period of 24 hrs.

\section{Mouse intestinal regeneration and IHC}

10-11-week-old male and female $C 57 B L / 6$ mice were used in these experiments. Mice were subject to whole body $10 \mathrm{~Gy}$ gamma-irradiation and the intestines were analysed $72 \mathrm{hrs}$ post-irradiation, which represents the proliferative phase of the regenerative response to damage in the mouse small intestine ${ }^{28}$. Small intestines were isolated and flushed with tap water. $10 \times 1 \mathrm{~cm}$ portions of small intestine were bound together with surgical tape and fixed in $4 \%$ neutral buffered formalin. Intestines from 3 mice per condition were used. $4 \mu \mathrm{m}$ sections of formalin-fixed paraffin-embedded (FFPE) tissues were cut, mounted onto adhesive slides and incubated at $60^{\circ} \mathrm{C}$ overnight. Prior to staining, sections were dewaxed for 5 minutes in xylene followed by rehydration through decreasing concentrations of alcohol and final washing with $\mathrm{H}_{2} \mathrm{O}$ for 5 minutes. FFPE sections underwent heat-induced epitope retrieval in a Dako pre-treatment module. Sections were heated in Target Retrieval Solution High pH (Dako, K8004) for 20 minutes at $97^{\circ} \mathrm{C}$ before cooling to $65^{\circ} \mathrm{C}$. Slides were removed and washed in Tris Buffered Saline with Tween (TbT) (Dako, K8000) and loaded onto a Dako autostainer link48 platform where they were stained with anti-CD31 antibody 1:75 (Abcam, ab28364) following standard IHC procedures. All animal work was approved by a University of Glasgow internal ethics committee and performed in accordance with institutional guidelines under personal and project licences granted by the UK Home Office to J.B.C (PPL PCD3046BA).

\section{Single terminal tracheal cell clones}

To generate single cell clones of terminal tracheal cells, parental lines were allowed to mate for 2-3 days, after which adults were moved into new vials and $\mathrm{F} 1$ progenies where heat shocked in a water bath for $1 \mathrm{hr}$ at $37^{\circ} \mathrm{C}$. Adults of the correct genotype, emerging from the heat shocked animals, were selected and aged at $25^{\circ} \mathrm{C}$ for 10 days followed by feeding with Sucrose (control) or $P e$ for $16 \mathrm{hrs}$ to cause intestinal damage and induce regeneration. Tissues were then dissected and processed for immunofluorescence staining and confocal imaging. 


\section{Hypoxia treatment}

Adult flies were aged at $25^{\circ} \mathrm{C}$ or $29^{\circ} \mathrm{C}$ in $21 \% \mathrm{O}_{2}$ (Normoxia) at a density of $15-20$ flies per vial. Then, animals were transferred overnight to $3 \% \mathrm{O}_{2}$ (Hypoxia) in a Whitley Scientific H35 hypoxystation incubator.

\section{Drosophila immunohistochemistry}

Immunohistochemistry was carried out as described previously ${ }^{32}$. The following antibodies were used: chicken anti-GFP 1:200 (Abcam, ab13970), mouse anti-PH3 1:100 (Cell Signaling, 9706), rabbit anti-Dcp1 1:100 (Cell Signaling, \#9578S), rabbit anti- $\beta$ gal 1:1000 (MP Biochemicals \#559761), rabbit anti-DsRed 1:1000 (Clontech, \#632496), mouse antiArm 1:3 (Hybridoma Bank, N2 7A1), mouse anti-Dlg 1:100 (Hybridoma Bank, 4F3), rabbit anti-p-Erk 1:100 (Cell Signaling, \#9101), guinea pig anti-Myc 1:100 (gift from Gines Morata) and rabbit anti-Trh 1:100 (gift from M. Llimargas). Chitin Binding Protein (CBP 1:100; gift from M. Llimargas) was used to visualise all tracheal tissue. Alexa Fluor 488, 594 and 647 (Invitrogen) were used as secondary antibodies labels at 1:200 and 1:100 respectively. Guts were mounted in Vectashield anti-fade mounting medium for fluorescence with DAPI (Vector Laboratories, Inc) to visualize all nuclei.

\section{Image acquisition}

Transmission Electron Microscopy-Guts were dissected under Schneider's insect medium and were subsequently fixed in $2.5 \%$ glutaraldehyde in $0.1 \mathrm{M}$ cacodylate buffer (pH 7.4) for $1 \mathrm{hr}$ at room temperature. Samples were rinsed repeatedly in $0.1 \mathrm{M}$ sodium cacodylate buffer, before fixation treatment in $1 \%$ Osmium Tetroxide/buffer for $1 \mathrm{hr}$, followed by washing with $\mathrm{dH} 20$ for 30mins. Samples were then stained in $0.5 \%$ Uranyl Acetate/dH20 for $1 \mathrm{hr}$ (in the dark) and dehydrated by incubation in a graded series of Ethanol. Samples were subject to three subsequent incubations in Propylene Oxide followed by Epon 812 resin/Propylene Oxide (50:50) mix and left on a rotator overnight, followed by several incubations in pure Epon resin. Samples were then embedded into blocks and oven incubated at $60^{\circ} \mathrm{C}$ for $48 \mathrm{hrs}$. Ultrathin sections (50-70nm thickness) were cut using a Leica Ultracut UCT. The sections produced were collected on Formvar coated 100 mesh copper grids and subsequently contrast stained in 2\% Methanolic Uranyl Acetate for $5 \mathrm{mins}$ followed by Reynolds Lead Citrate for 5mins. Gut samples were viewed using a JEOL 1200 EX TEM and Images captured using a Cantega $2 \mathrm{~K}$ x $2 \mathrm{~K}$ camera and Olympus iTEM-TEM imaging platform.

Confocal microscopy (Zeiss LSM 780)—Each image represents half-volume of the full posterior midgut (area comprised between hindgut and Copper Cell Region) and were acquired with 20x, 40x or 63x lenses using identical acquisition conditions for all samples from a given experiment. Images represent maximal intensity projection of a stable number of Z-Stacks and were processed with ImageJ and Carl Zeiss Zen 3.0 to adjust brightness and contrast.

Light microscopy (Axio observer Zeiss)—Adult guts were dissected in PBS, mounted in $100 \%$ glycerol and imaged immediately. Up to three pictures per posterior midgut were taken to cover most of the area. Images were taken at their most apical plane to best detect 
TTCs with a 20x lens. Images from mouse intestinal samples (Fig. 1n) were acquired under a 20x lens using this microscope and were processed with ImageJ and Carl Zeiss Zen 2.0.

\section{Quantifications in the adult posterior midgut}

Quantification of ISC proliferation-Antibodies against Phosphorylated Histone H3 (PH3) were used to detect ISC proliferation in the adult midgut. The total number of $\mathrm{PH}^{+\mathrm{ve}}$ cells per posterior midgut was quantified manually upon visual inspection using an Olympus BX51 microscope. The number of midguts analysed (n) for each experiment are indicated in the figures.

Quantification of tracheal coverage from immunofluorescence images-Unless otherwise noted, confocal images of $d S R F>G F P$ expressing midguts were used and tracheal values provided were obtained from quantification of tile scan images of the entire R4-R5 posterior midgut regions, acquired with a 20x lens. Values of tracheal coverage represent pixel per area and were obtained from maximum intensity Z-projections. Pictures were individually processed on ImageJ as follows: 1) maximum intensity projection from $\mathrm{Z}$ stacks were produced; 2) area of interest was cropped to eliminate Malpighian tubules, hindgut and copper cell region; 3) "threshold" was adjusted to ensure the detection of most of terminal tracheal branches; 4) function "skeletonize" was applied to generate a skeleton of the tracheal network; 5) maximum intensity of this skeleton was measured (Extended data Fig.1a). The number of posterior midguts analysed (n) for each experiment are indicated in the figures.

Quantification of tracheal branching form light-microscopy images-Acquired images were blindly scored using a 1 to 5 scoring system (Extended Data Fig.3a, c). The custom ImageJ macro used for blind tracheal scoring is "Blind_scoring.ijm". Between 1 and 3 images were acquired for each posterior midgut. The number of midguts analysed (n) for each experiment are indicated in the figures.

Quantification of total branches per TTC and TTC ramifications-Maximum intensity projections from confocal $\mathrm{Z}$ stacks were used. The number of primary, secondary and tertiary branches derived from individual TTCs was assessed (Fig.1i). Due to the intrinsic complexity of the tracheal, it is difficult to unambiguously assign cellular extensions/branches to a single TTC. To circumvent this issue, we counted tracheal branches starting from a TTC body and defined the end of a TTC extension when it touched the body of another TTC. Additionally, the generation of single TTC clones allowed us to unambiguously quantify the total number of branches from individual TTC, which we did manually (Fig.1f, h). These two approaches led to same outcome. Number of TTC (n) and midguts analysed for each condition are indicated in the corresponding figures and figure legends.

Quantification of total tracheal length-We used the plugin "NeuronJ" from ImageJ to quantify the total length of all branches emerging from a TTC (Fig.1k). Number of TTC (n) and midguts analysed for each condition are indicated in the corresponding figures and figure legends. 
Quantification of TTC nuclei-Confocal images of posterior midguts from animals expressing $d S R F^{t s}>\operatorname{RedStinger~to~label~TTC~nuclei~were~used.~The~custom~ImageJ~macro~}$ used for quantifying the number of TTC nuclei is "dSrf_pH3_overlap_for Jessica.ijm". This macro was also used to quantify the number of TTC nuclei positive for $\mathrm{PH}^{+}$staining (Extended data Fig.1g). Number of midguts analysed (n) for each condition is indicated in the corresponding figure and figure legend.

Quantification of posterior midgut area-Midgut tissue was visualized by DAPI staining and the posterior midgut area (length $\mathrm{x}$ width) was measured with ImageJ (Extended Data Fig.1c, d). Number of midguts analysed (n) for each condition is indicated in the corresponding figure and figure legend.

Quantification of lacZ reporters-Antibodies against $\beta$-galactosidase were used to detect lactate dehydrogenase-, Delta-, breathless-, bs/dSRF-, branchless- and trachealesslac $Z$ reporters. Pictures were taken with confocal microscopy and staining was quantified using ImageJ. For each gut quantified, the background staining signal was subtracted from the total signal of $\beta$-galactosidase detected in TTCs, ISCs/EBs or ECs. This value was then divided by the background signal to normalize the data. Number of cells (n) and midguts analysed for each condition are indicated in the corresponding figures and figure legends.

Quantification of cell death-Antibodies against Dcp1 were used to assess cell death in posterior midguts. Pictures were taken with confocal microscopy and Dcp1 staining intensity was measured relative to the surface of the gut area analysed. Number of midguts analysed (n) for each experiment are indicated corresponding in the figure and figure legend.

\section{Quantification of pERK, Imp::GFP, Myc and Trachealess staining-Midguts}

stained with antibodies to detect these proteins included a methanol fixation step between the PFA fixation and PBST washing steps of the standard protocol, as described previously ${ }^{41}$. Images were acquired with confocal microscopy and staining was quantified using ImageJ. For each gut quantified, the background staining signal was subtracted from the total antibody signal within DAPI positive cells. This value was then divided by the background signal in order to normalize the data. The number of cells (n) and midguts analysed for each condition are indicated in the corresponding figures and figure legends.

\section{RT-qPCR}

Trizol (Invitrogen) was used to extract total RNA from 30 midguts per biological replicate. cDNA synthesis was performed using the High-Capacity cDNA Reverse Transcription Kit (Applied Biosystems). QuantiNova SYBR Green (Qiagen) was used for qPCR. Samples were run in a QuantStudio 3 Real-Time PCR Systems. Data were extracted and analysed using and QuantStudio ${ }^{\text {TM }}$ Design and Analysis desktop Software v1.4.3 and Prism 6.07. Data from 5 biological replicates is presented as the mean fold change with S.E.M. Expression of target genes was measured and normalized to gapdh1 or act5c using standard curves. Primer sequences can be found in Supplementary Table 4. 


\section{Targeted DamID ( $\mathrm{TaDa})$, library preparation, sequencing and data analysis}

dSRF-GAL4; tub-gal8O ${ }^{\text {ts }}$ (dSRF's ) animals were crossed to UAS-LT3-Dam or UAS-LT3-

Dam-Pol II animals at $18^{\circ} \mathrm{C}$. F1 progeny were collected every $48 \mathrm{hrs}$ and aged for a further 7 days at $18^{\circ} \mathrm{C}$ before transferring to $29^{\circ} \mathrm{C}$ to induce adult restricted Dam protein expression for $24 \mathrm{hrs}$. During the last $16 \mathrm{hrs}$ at $29^{\circ} \mathrm{C}$, flies were fed a Sucrose or Sucrose $+P e$ solution. 60 midguts per condition per biological replicate were dissected in cold PBS and stored at $-80^{\circ} \mathrm{C}$. Methylated DNA fragments were isolated and next generation sequencing libraries were prepared as described previously ${ }^{46}$. Sequencing data from TaDa experiments were processed using a previously described pipeline ${ }^{62}$ and mapped to release 6.03 of the Drosophila genome. Transcribed genes were annotated for Pol II binding data using a custom Perl script ${ }^{47}$ and release 6.11 of the annotated Drosophila genome. Monte Carlo simulations were performed using randomised data to generate FDR. Genes with significant RNA pol II binding were identified based on meeting a threshold of $1 \%$ FDR and $>0.2 \log 2$ ratios. Briefly, a $\log 2$ ratio of the Dam-RNA Pol II read counts over control Dam-only read counts is calculated after quantile normalisation ${ }^{62}$ and if this ratio is higher than 0.2 , then we would determine that this gene has significant RNA Pol II binding (Supplementary Table 1). Significance was assigned based on the signal from multiple GATC fragments and using a very stringent pipeline as a transcript had to have a false discovery ratio (FDR) of less than $1 \%$ in both replicates to be called significant.

Gene Ontology (GO) term analysis was performed using the "enrichGo" function of R package ClusterProfiler ${ }^{63}$ to search for enriched GO terms (Supplementary Table 2). Three independent biological replicates were originally processed for each condition (Sucrose and $P e)$. However, after visual inspection of the sequencing tracks, one replicate from each condition was excluded from the analysis, due to poor DNA sample quality and unreliable sequencing data. Scattered plots showing the correlation between samples for each condition are provided (Extended data Fig.7a).

\section{Statistics and Reproducibility}

Most experiments represent between 2 and 3 independent biological replicates with similar results. RT-qPCRs were done using 5 independent biological replicates. Data in Figs. 1d, c; 3g, h; 4a, d; 7c and Extended data Figs. 2a, d; 3b, c; 4d; 5a represent single biological replicate experiments with either multiple sets of samples and/or that have been repeated in other contexts throughout the manuscript. Each biological replicate represents a set of control and experimental tissues processed within the same day and belonging to animals derived from cultures with controlled feeding conditions, developmental timing and population density. Each biological replicate was processed in different days, and derives from independent F1s, which have been reared in independent batches of food.

GraphPad Prism version 6.07 (GraphPad Software) was used for statistical analyses. To compare two groups, we used two-tailed Student's t-test. To compare multiple groups, we used two-way ANOVA and Sidak's multiple comparison test. NS, not significant ( $p>0.05$ ); ${ }^{*} \mathrm{p}<0.05,{ }^{* *} \mathrm{p}<0.01, * * * \mathrm{p}<0.001 * * * * \mathrm{p}<0.0001$. Further information on sample size, statistical tests used and $P$ values for each experiment are indicated in figure legends. 


\section{Extended Data}
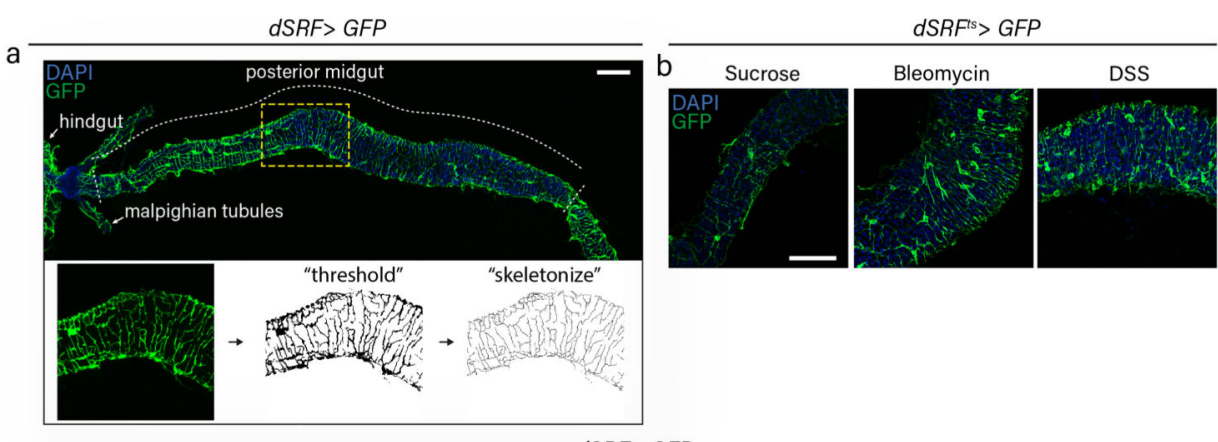

C

$d S R F>$ GFP
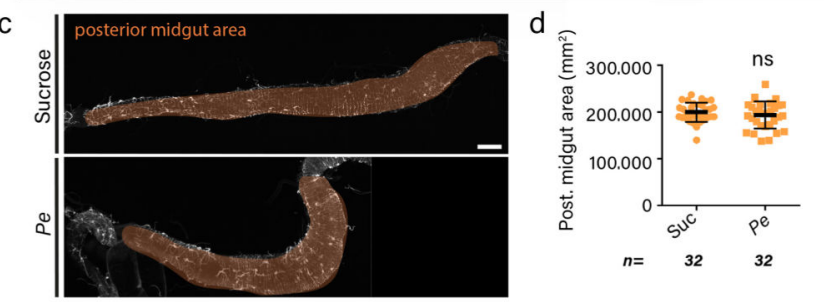

e
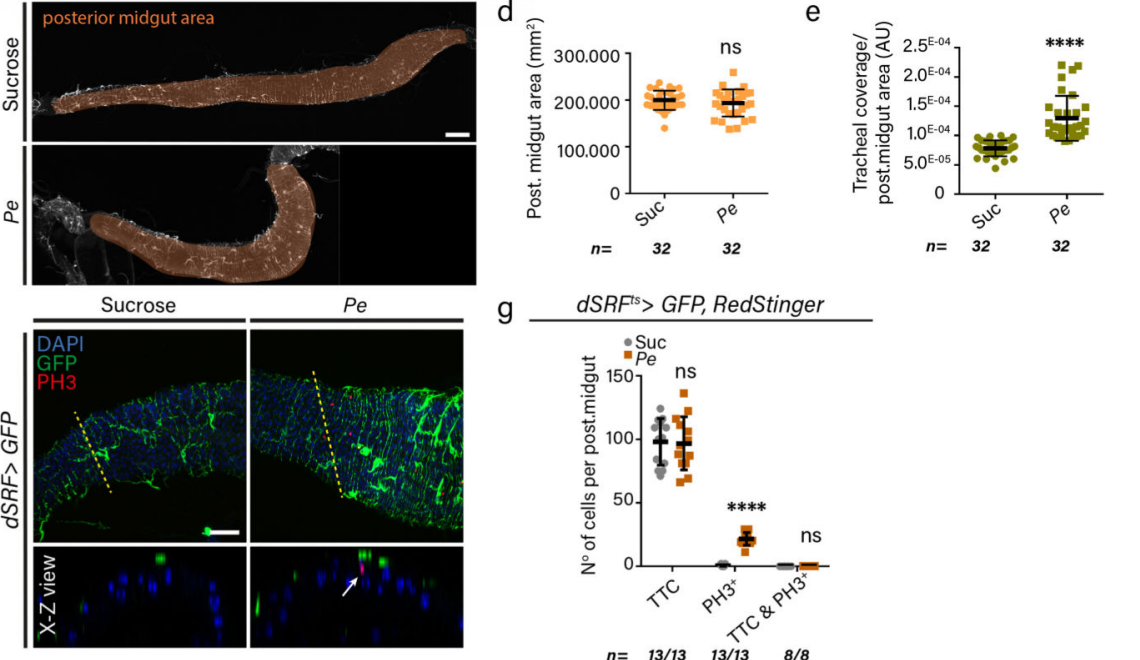

g $d S R F^{s s}>G F P$, RedStinger

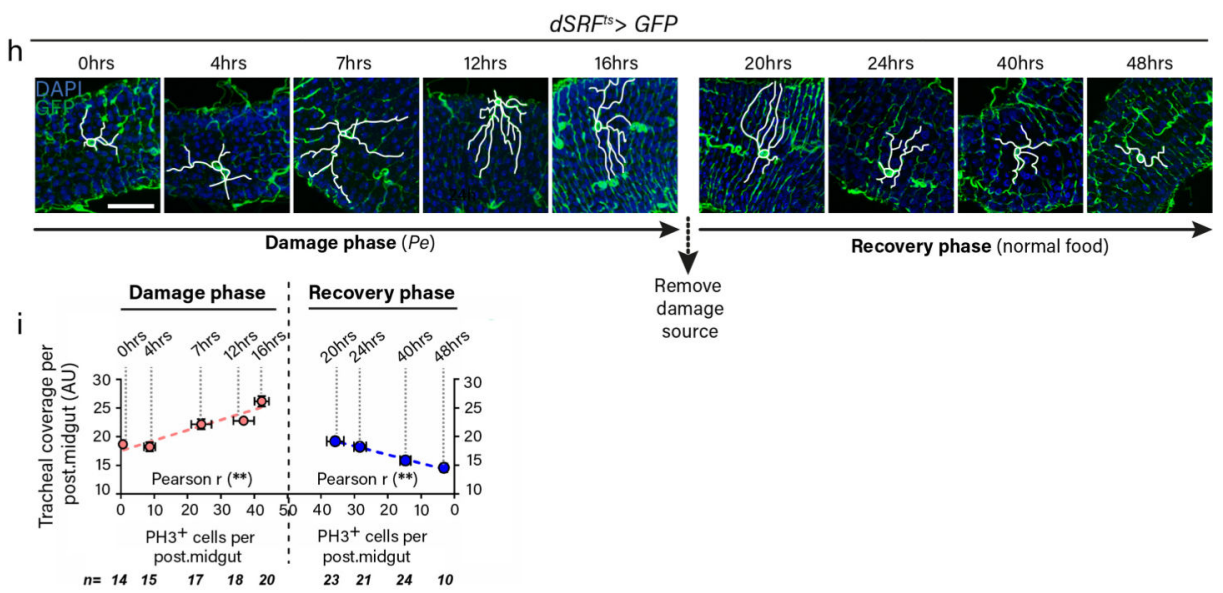

Extended Data Fig. 1. Characterization and quantification of adult tracheal remodelling following intestinal damage

a, (top), Adult posterior midgut and associated TTCs (green). (bottom), illustration of the different steps followed for the quantification of gut tracheal coverage as explained in Methods. Scale bar: $100 \mu \mathrm{m}$. Box in top panel highlights the area shown in the bottom panels b, Confocal images of TTCs (green) from posterior midguts of control animals (Suc) or animals fed with Bleomycin or DSS. Scale bar: $50 \mu \mathrm{m}$. c, Representative confocal images of 
Sucrose or Pe treated midguts (shaded in brown). Scale bar: $100 \mu \mathrm{m}$. d, e, Quantification of posterior midgut area (d) and ratio of tracheal coverage over adult posterior midgut area (e). Two-tailed, unpaired T-test; $\mathrm{n}=$ number of posterior midguts, indicated in panels. d, $P=0.3464$. e, $(* * * * P<0.0001)$. f, Confocal images of TTCs (green) from Sucrose or $P e$ treated midguts stained with anti-PH3 to label proliferating ISCs (red). Bottom panels represent orthogonal views of the midguts shown in top panels. Scale bar: $50 \mu \mathrm{m}$. g, Quantification of individual or combined TTC nuclei and $\mathrm{PH}^{+}$cells in control or $\mathrm{Pe}$ infected midguts. Two-tailed, unpaired T-test $(* * * * P<0.0001) ; \mathrm{n}=$ number of posterior midguts, indicated in panel. $\mathbf{h}$, Confocal images of adult TTCs assessed at the indicated time points during and after intestinal damage. White lines trace individual TTCs. Scale bar: $50 \mu \mathrm{m}$. i, Correlation graph between TTC coverage and ISC proliferation for each of the time points and conditions presented in (h). $n=$ number of posterior midguts, indicated in panels. Pearson's correlation coefficient (damage $r=0.9660$, recovery $r=$ recovery 0.9962 , damage $* * P=0.0075$, recovery $* * P=0.0038)$. Values represent mean \pm S.E.M. 

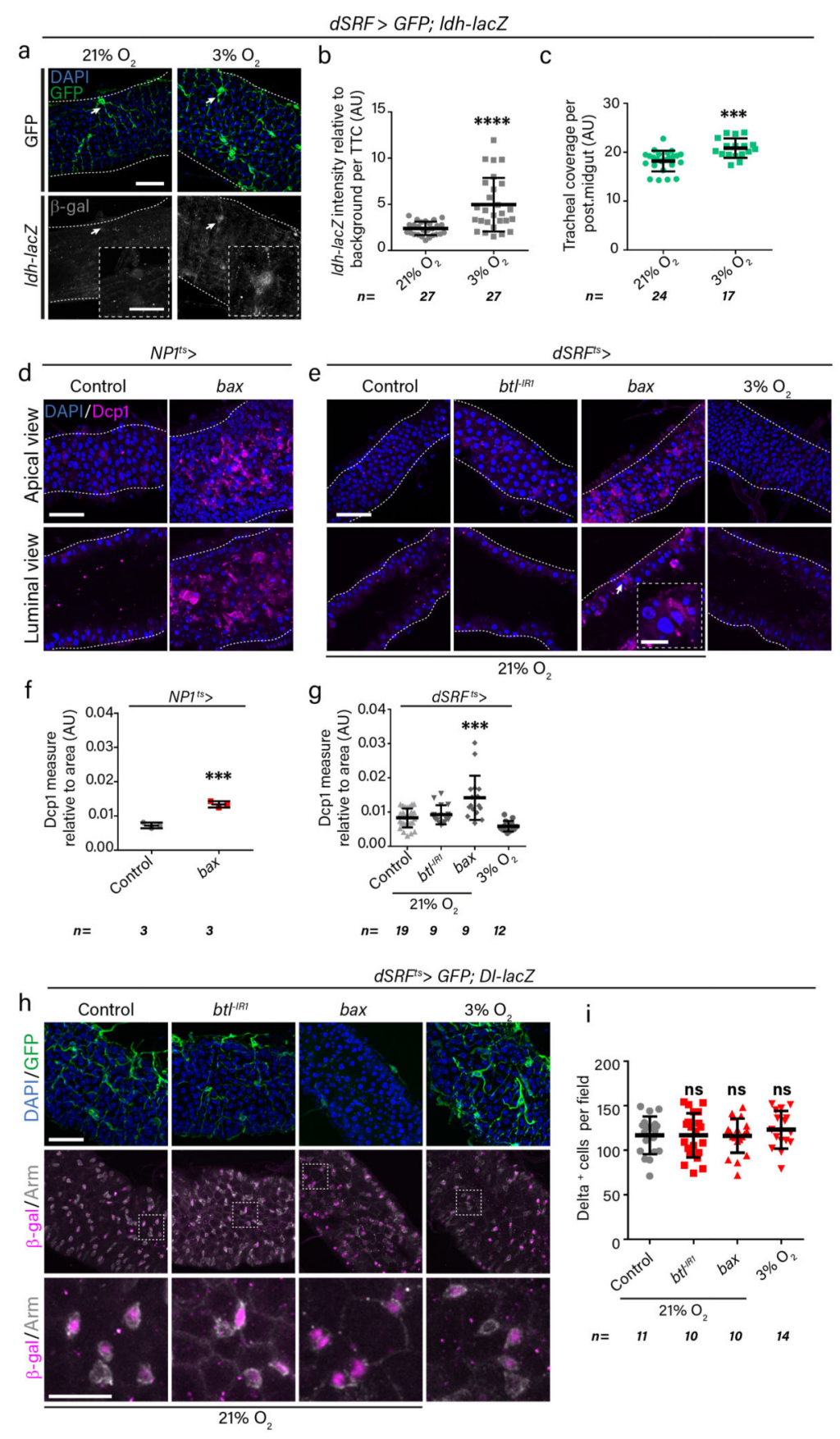

Extended Data Fig. 2. Hypoxia or impaired TTC remodelling does not affect ISC survival. a, TTCs (green) and the Sima/HIF-1a activity reporter $1 d h-l a c Z$ (grey) in adult posterior midguts from animals in normoxia $\left(21 \% \mathrm{O}_{2}\right)$ or subjected to $24 \mathrm{hrs}$ of hypoxia $\left(3 \% \mathrm{O}_{2}\right)$. Dotted boxes show a magnified view of TTCs pointed by arrows. Scale bars: $50 \mu \mathrm{m}$ (main figure); $20 \mu \mathrm{m}$ (close up view). b, ldh-lac $Z$ staining intensity within TTCs, relative to background, in midguts as in (a). Two-tailed, unpaired T-test $(* * * * P<0.0001)$; $\mathrm{n}=$ number of TTCs from 9 posterior midguts per condition, indicated in panels. c, Tracheal coverage in adult posterior midguts as in (a). Two-tailed, unpaired T-test ( $* * * P=0.0003)$; $\mathrm{n}=$ number of 
posterior midguts, indicated in panels. d, e, Midguts stained with anti-Dcp1 (magenta) to visualize cell death in control animals; upon adult-specific bax overexpression in ECs (d); animals subjected to hypoxia or to indicate the genetic TTC disruptions (e). d, e, Upper panels (apical tissue views); lower panels (longitudinal sections showing intestinal tube

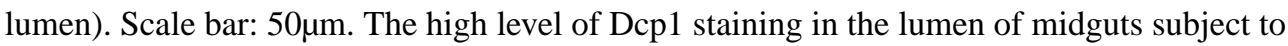
bax overexpression in ECs (d) corresponds to delaminating/dying cells. Dotted box in (e) shows a magnified view of an apoptotic EC pointed by arrow in main figure and identified

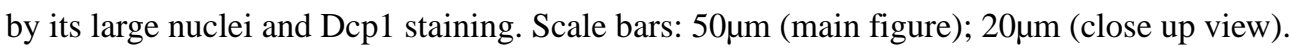
$\mathbf{f}, \mathbf{g}$, Quantification of Dcp1 staining in midguts as in (d, e). Two-tailed, unpaired T-test; $\mathrm{n}=$ number of posterior midguts, indicated in panels. $\mathbf{f},(* * * P=0.0009)$. $\mathbf{g}$, ( bax vs Control, $* * * P=0.0003$ ). h, TTCs (green) and ISCs detected with a Delta-lac $Z$ reporter (magenta) and anti-Armadillo (Arm) staining (grey) in midguts as in (e). Dotted boxes in middle panels indicate the magnified areas in the lower panels. Scale bars: $50 \mu \mathrm{m}$ (main figure); $20 \mu \mathrm{m}$ (close up view). i, Number of Delta-lacZ positive cells in midguts as in (h). Two-tailed, unpaired T-test; $n=$ number of midguts, indicates in panels. Values represent mean \pm S.E.M. 
a
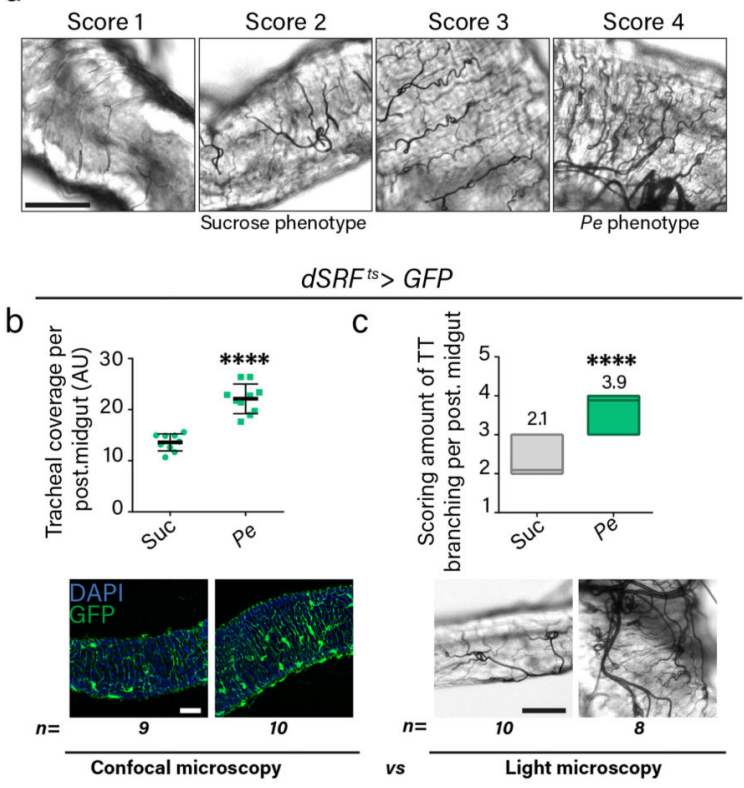

$N P 1^{t s}>$

d

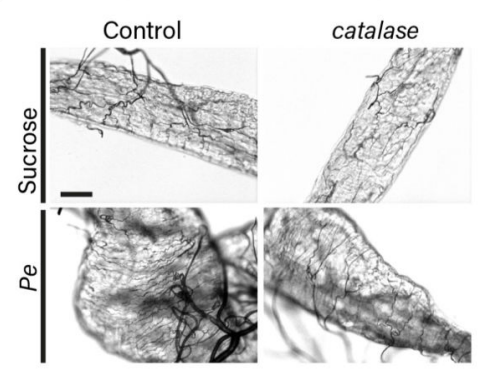

e

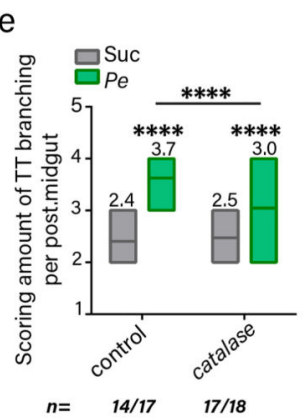

f

g

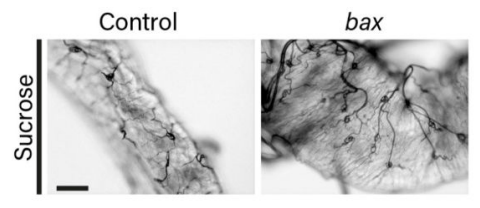

$\mathrm{h}$ 。

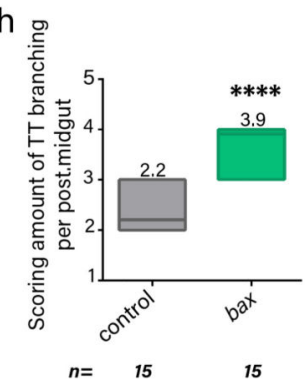

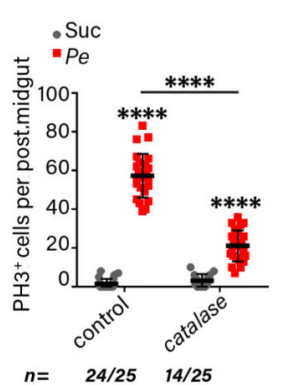

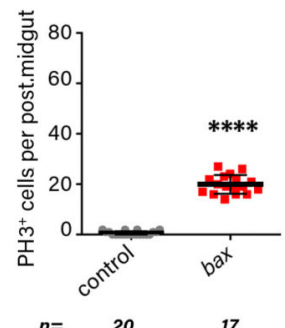

Extended Data Fig. 3. Gut associated TTCs are responsive to local signals from the damaged intestinal epithelium.

a, Examples of tracheal branching levels assigned to each of the scores used for the quantification of tracheal coverage from light microscopy images. Scale bar: $50 \mu \mathrm{m}$. b, c (top panels), Quantification of tracheal branching from confocal (b) and brightfield images (c) of Sucrose or Pe treated midguts. Two-tailed, unpaired T-test $(* * * * P<0.0001)$; $\mathrm{n}=$ number of posterior midguts, indicated in panels. b, c (bottom panels) Representative confocal (b) or brightfield images (c) from midguts as in top panels. Scale bars: $50 \mu \mathrm{m}$. d, Brightfield images 
from Sucrose or Pe treated midguts from wild type (control) animals or upon catalase

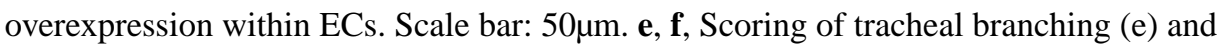
quantification of $\mathrm{PH}^{+}$ISCs (f) in posterior midguts as in (d). Two-way ANOVA followed by Sidak's multiple comparisons test $(* * * * P<0.0001) ; \mathrm{n}=$ number of midguts quantified, indicated in panels. $\mathbf{g}$, Brightfield images of posterior midgut from control animals or upon adult-specific overexpression of bax in ECs. Scale bar: $50 \mu \mathrm{m}$. h, i, Quantification of tracheal branching (h) and $\mathrm{PH}^{+}{ }^{+} \mathrm{ISCs}$ (i) in posterior midguts as in (g). Two-tailed, unpaired T-test $(* * * * P<0.0001) ; \mathrm{n}=$ number of midguts, indicated in panels.

Box plots represent maxima, minima and mean. Mean value is indicated on top of boxes. Otherwise, values represent mean \pm S.E.M.

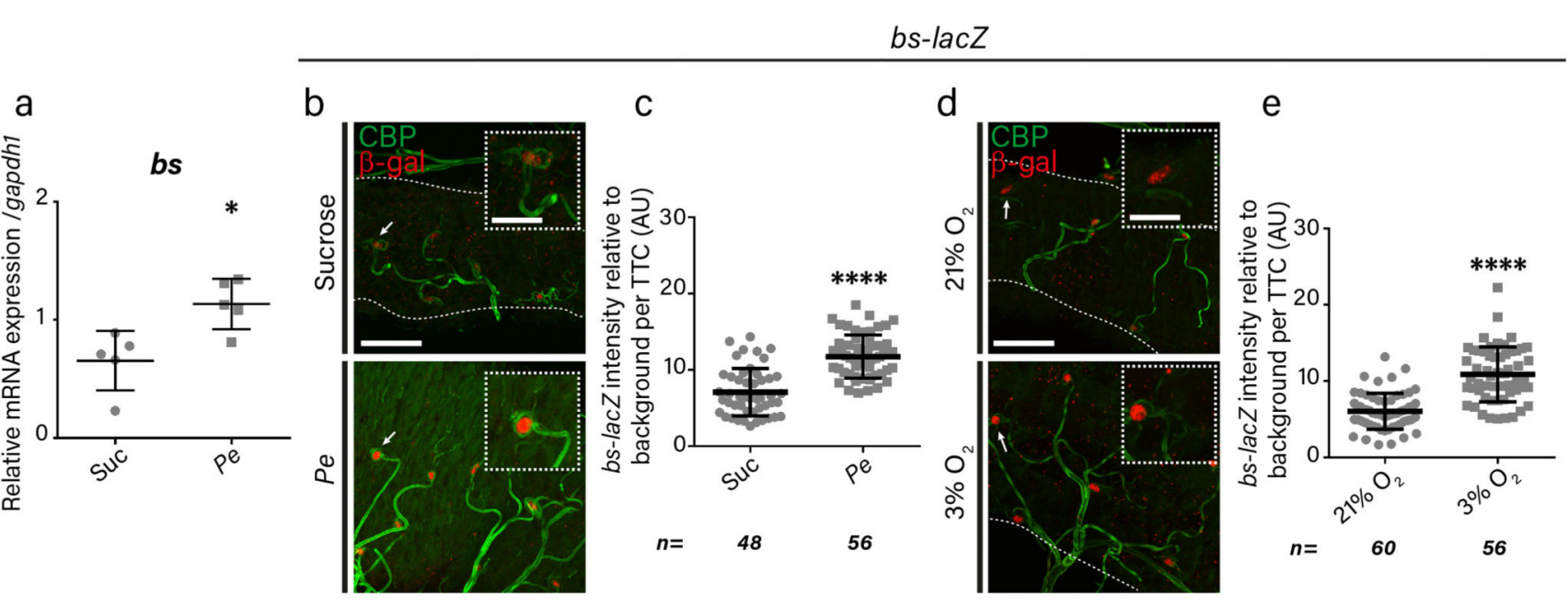

$d S R F^{t s}>$ GFP

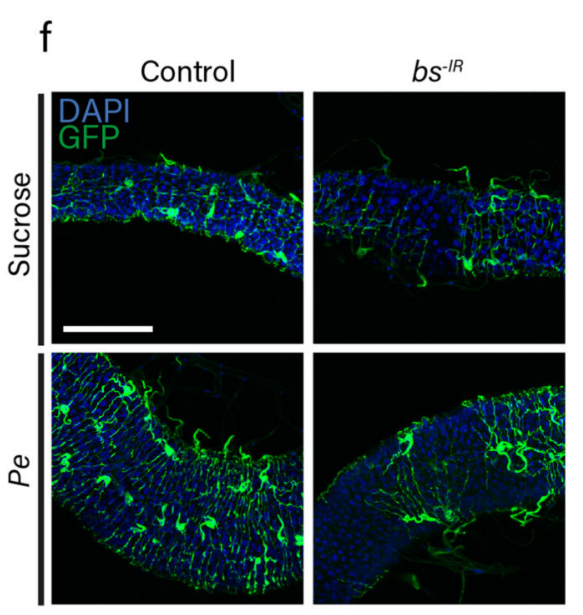

g

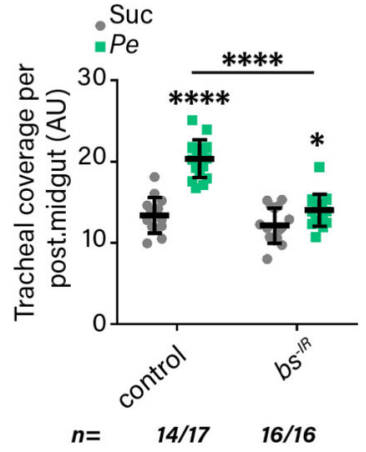

h

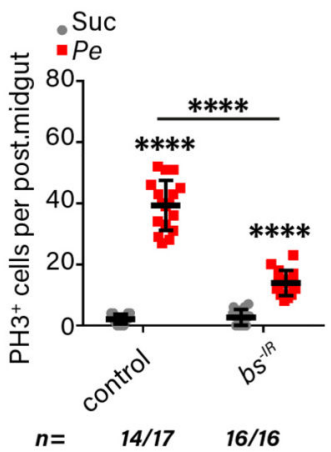

Extended Data Fig. 4. blistered/dSRF expression is upregulated in adult TTCs following intestinal damage or hypoxia and it regulates damage induced TTC remodelling and ISC proliferation. a, qRT-PCR for blistered ( $b s) / d S R F$ mRNA expression from adult midguts and associated tracheal tissue, in control (Sucrose) or Pe treated midguts. Two-tailed, unpaired T-test $(* P=0.0117) ; \mathrm{n}=5$ biological replicates. $\mathbf{b}$, Confocal images of $b s$-lac $Z$ reporter expression 
(red) in Sucrose and Pe treated midguts stained with Chitin Binding Protein (CBP, green) to visualize all tracheal tissue. Dotted boxes show a magnified view of TTCs pointed by

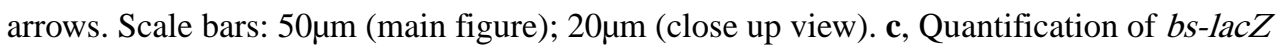
staining intensity within TTCs in posterior midguts as in (b). Two-tailed, unpaired T-test (**** $P<0.0001$ ); $\mathrm{n}=$ number of TTCs from 12-15 midguts per condition, indicated in panel. d, Confocal images of $b s-l a c Z$ reporter expression (red) in adult posterior midguts from animals housed in normoxia $\left(21 \% \mathrm{O}_{2}\right)$ or subjected to $16 \mathrm{hrs}$ of hypoxia $\left(3 \% \mathrm{O}_{2}\right)$ and stained with CBP (green). Dotted boxes show a magnified view of TTCs pointed by arrows. Scale bars: $50 \mu \mathrm{m}$ (main figure); $20 \mu \mathrm{m}$ (close up view). e, Quantification of $b s$-lac $Z$ staining intensity within TTCs in posterior midguts as in (d). Two-tailed, unpaired T-test (****P< 0.0001); $\mathrm{n}=$ number of TTCs from 12-15 midguts per condition, indicated in panel. $\mathbf{f}$, Confocal images of Sucrose and $P e$ treated midguts from wild type (control) animals or following $R N A i$-driven $b s$ knockdown $\left(b s^{-I R}\right)$ within TTCs. Scale bar: $100 \mu \mathrm{m} . \mathbf{g}, \mathbf{h}$, Quantification of tracheal coverage (g) and $\mathrm{PH}^{+}$ISCs in posterior midguts as in (f). Twoway ANOVA followed by Sidak's multiple comparisons test. $\mathbf{g},(* * * *<0.0001, * P<0.05)$. $\mathbf{h},(* * * * P<0.0001) ; \mathrm{n}=$ number of posterior midguts quantified, indicated in panels. Values represent mean \pm S.E.M. 

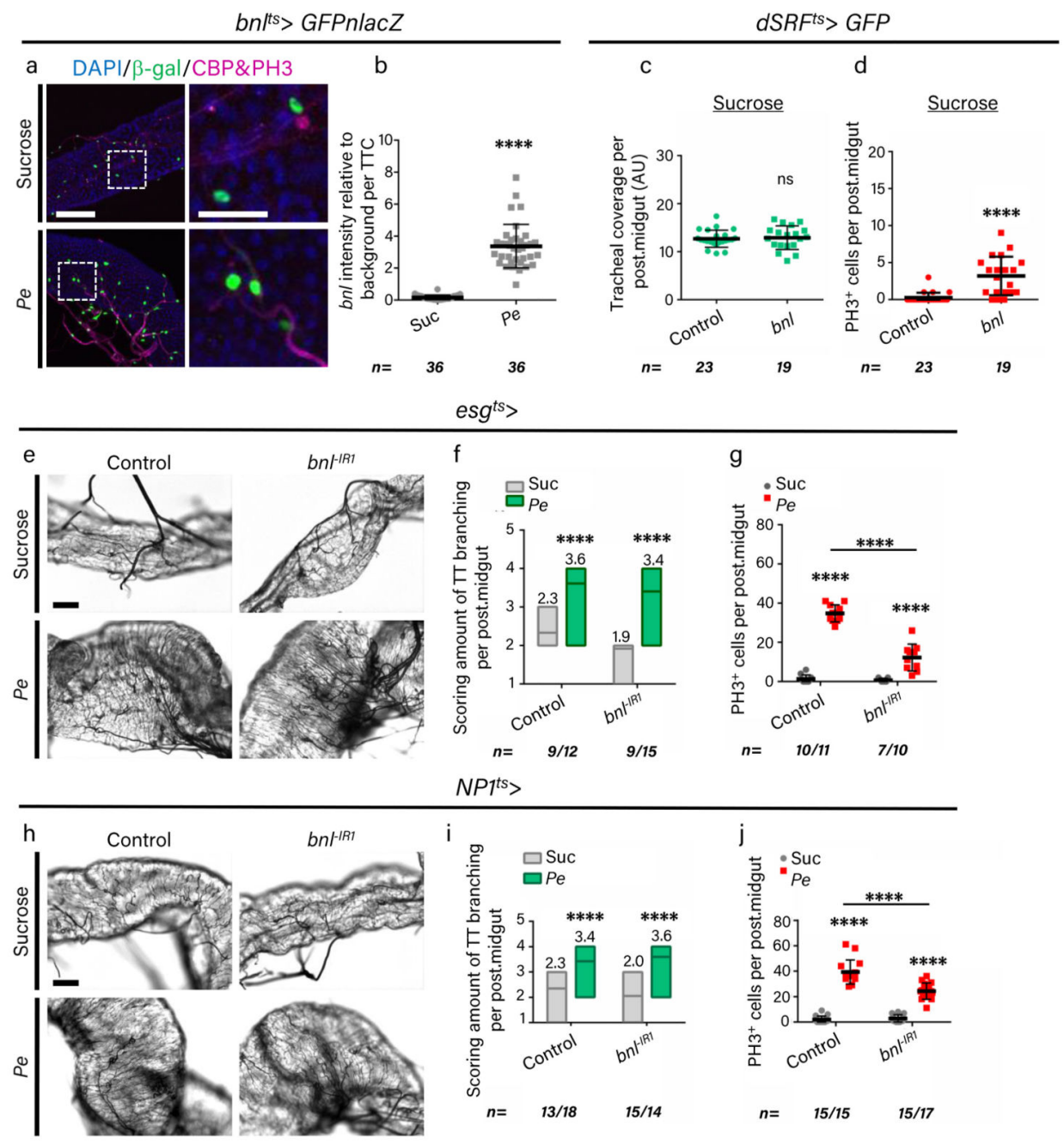

$N P 1^{t s}>$
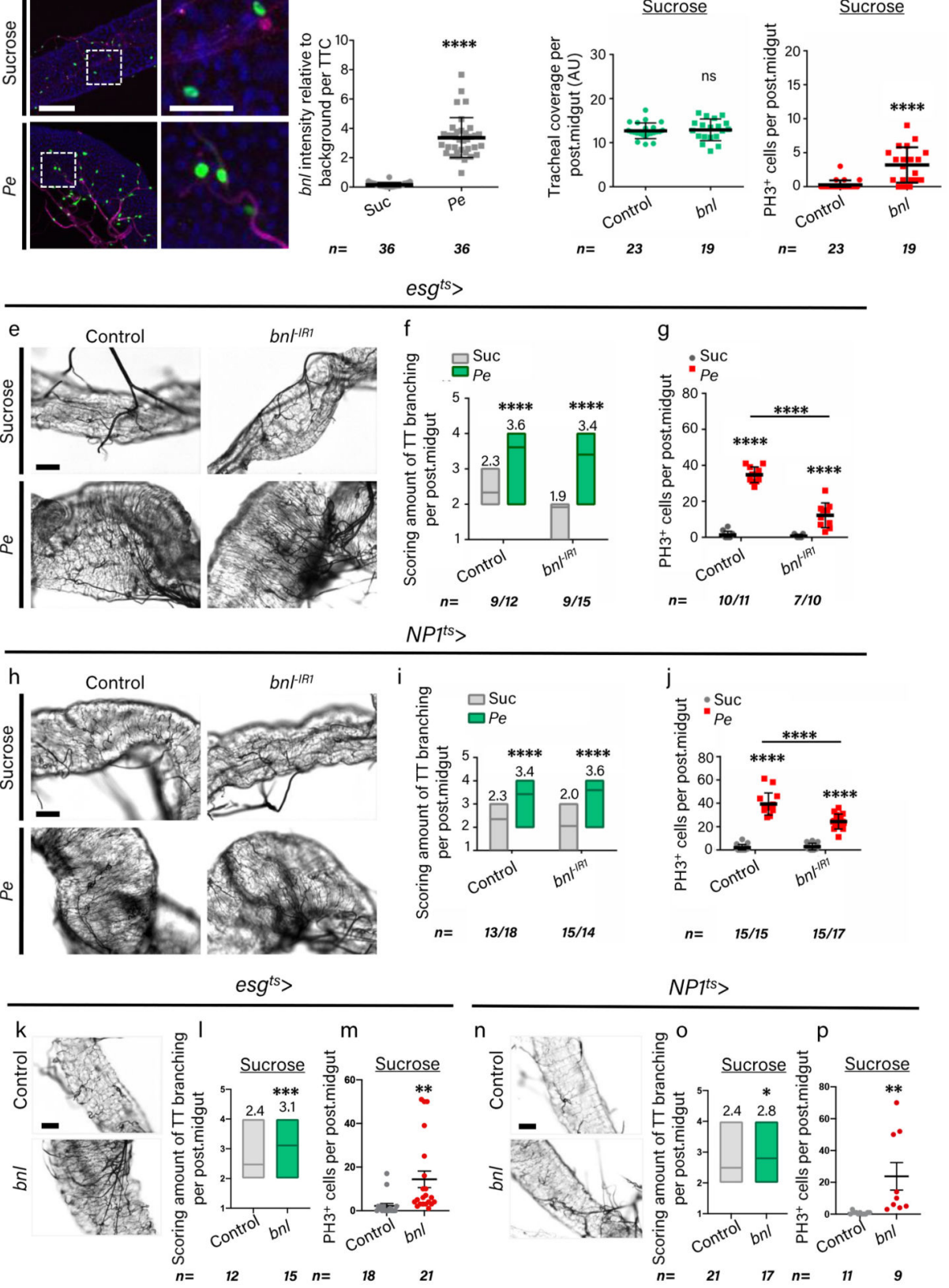

Extended Data Fig. 5. Multiple sources of Bnl individually contribute to regenerative ISC proliferation in the adult Drosophila midgut, independently of tracheal remodelling.

a, Confocal images of $F G F / b n l$ reporter expression bnl>GFPnlacZ (green) in Sucrose and $P e$ treated midguts stained with CBP (magenta) to visualise all tracheal tissue and PH3 (magenta). Dotted boxes in left panels indicate the area magnified in the right panels. Scale bars: $100 \mu \mathrm{m}$ (main figure); 40 $\mu \mathrm{m}$ (close up view). b, bnl>GFPnlacZ staining intensity relative to background within TTCs in midguts as in (a). Two-tailed, unpaired T-test $(* * * * P<0.0001) ; \mathrm{n}=$ number of TTCs from 12 midguts per condition, indicated in panel. $\mathbf{c}$, 
d Tracheal coverage (c) and $\mathrm{PH}^{+}$ISCs (d) from wild type (control) animals or upon bnl overexpression within TTCs. Two-tailed, unpaired T-test $(* * * * P<0.0001) ; \mathrm{n}=$ number of midguts, indicated in panels. e, Brightfield images from Sucrose or Pe treated midguts from wild type (control) animals or following RNAi-driven $b n l$ knockdown $\left(b n I^{I R}\right)$ within ISCs/

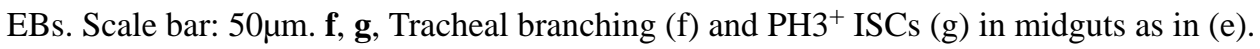
Two-way ANOVA followed by Sidak's multiple comparisons test $(* * * * P<0.0001) ; \mathrm{n}=$ number of midguts, indicated in panels. h, Brightfield images from Sucrose or $P e$ treated midguts from wild type (control) animals or upon $b n l$ knockdown $\left(b n I^{I R}\right)$ within ECs. Scale bar: 50 $\mu \mathrm{m}$. i, j, Tracheal branching (i) and $\mathrm{PH}^{+}{ }^{+}$ISCs (j) in midguts as in (h). Two-way ANOVA followed by Sidak's multiple comparisons test (**** $P<0.0001)$; $n=$ number of midguts, indicated in panels. $\mathbf{k}, \mathbf{n}$, Brightfield images of midguts from wild type (control)

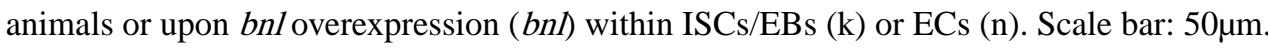
$\mathbf{l}, \mathbf{m}, \mathbf{o}, \mathbf{p}$, Tracheal coverage $(1, o)$ and $\mathrm{PH}^{+}$ISCs $(\mathrm{m}, \mathrm{p})$ from midguts as in $(\mathrm{k}, \mathrm{n})$. Twotailed, unpaired T-test; $\mathrm{n}=$ number of midguts, indicated in panels. $\mathbf{l},(* * * P=0.0002)$. $\mathbf{m}$, (** $P=0.0066) . \mathbf{o},(* P=0.0213) . \mathbf{p},(* * P=0.0084)$.

Box plots represent maxima, minima and mean. Mean value is indicated on top of boxes. Otherwise, values represent mean \pm S.E.M. 

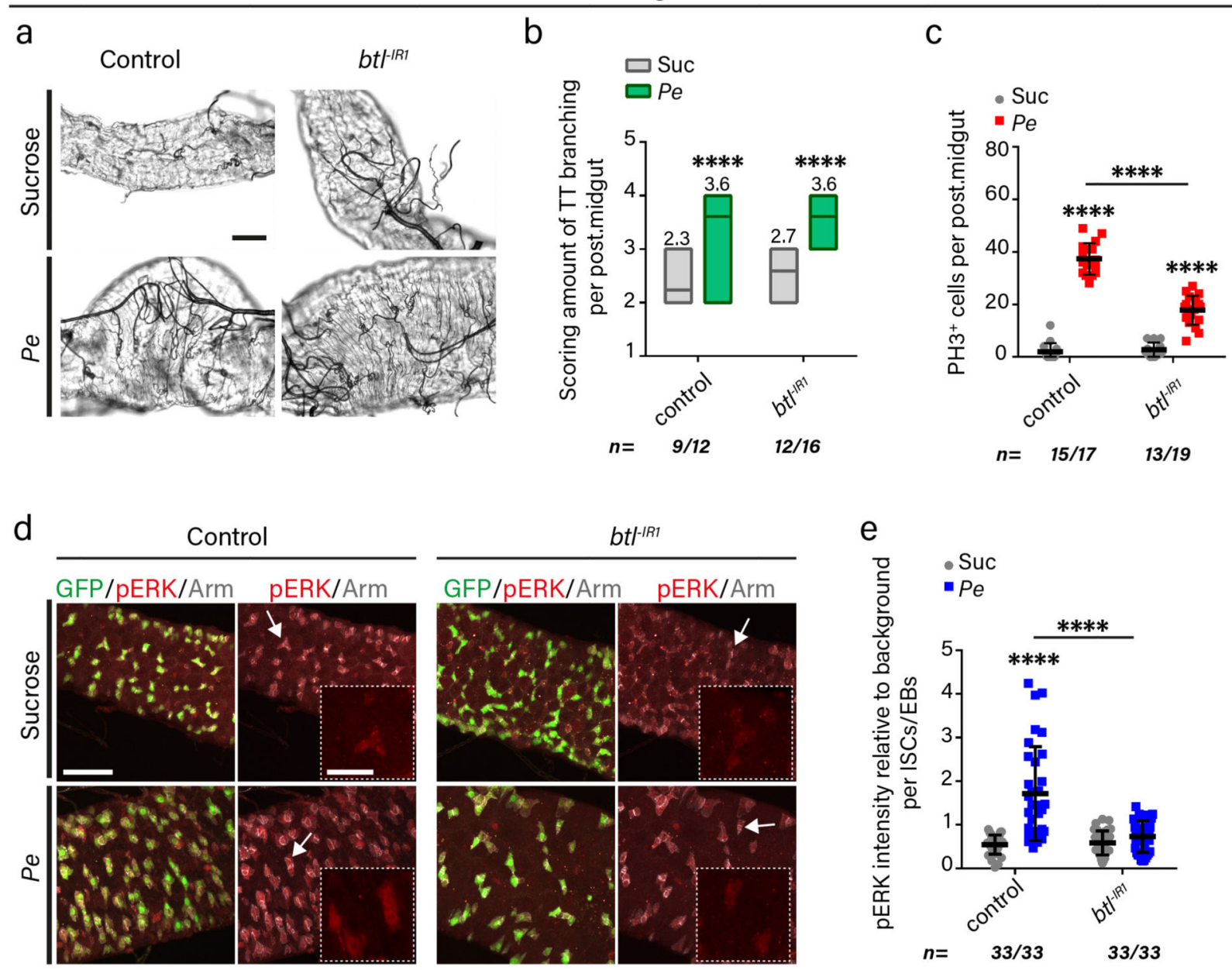

Extended Data Fig. 6. Individual sources of gut-derived Bnl are redundant for TTC remodelling but required for damage induced ISC proliferation

a, Brightfield images of Sucrose or Pe treated midguts from wild type (control) animals or upon RNAi-driven $b t l$ knockdown $\left(b t t^{I R}\right)$ within ISCs/EBs. Scale bar: $50 \mu \mathrm{m}$. b, c,

Quantification of tracheal branching (b) and $\mathrm{PH}^{+}$ISCs (c) in posterior midguts as in (a).

Two-way ANOVA followed by Sidak's multiple comparisons test (**** $P<0.0001)$; $=$ number of posterior midguts, indicated in panels. d, Representative confocal images of activated MAPK (pERK) staining (red), Arm (grey) and ISCs/EBs (esg $>G F P$, green) in control (Sucrose) or regenerating $(P e)$ adult posterior midguts from wild type (control) animals or upon RNAi-driven $b t l$ knockdown $\left(b t r^{I R}\right)$ within ISCs/EBs. Dotted boxes show a

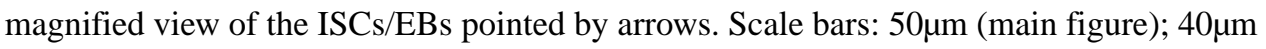
(close up view). e, Quantification of pERK staining intensity relative to background within ISCs/EBs in posterior midguts as in (d). Two-way ANOVA followed by Sidak's multiple comparisons test $(* * * * P<0.0001)$; $\mathrm{n}=$ number of ISCs/EBs from 11 posterior midguts per condition, indicated in panel.

Box plots represent maxima, minima and mean. Mean value is indicated on top of boxes.

Otherwise, values represent mean \pm S.E.M. 
a

Pearson correlation of average scores per genes

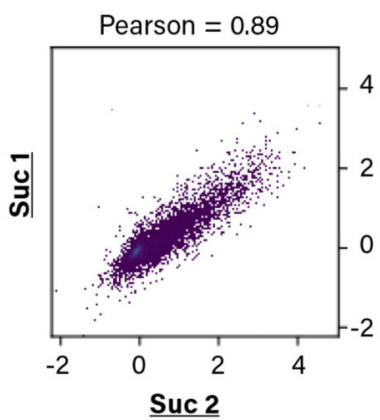

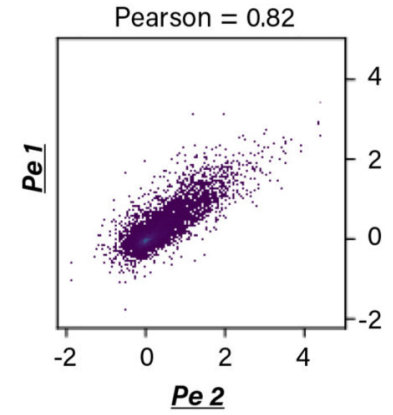

b

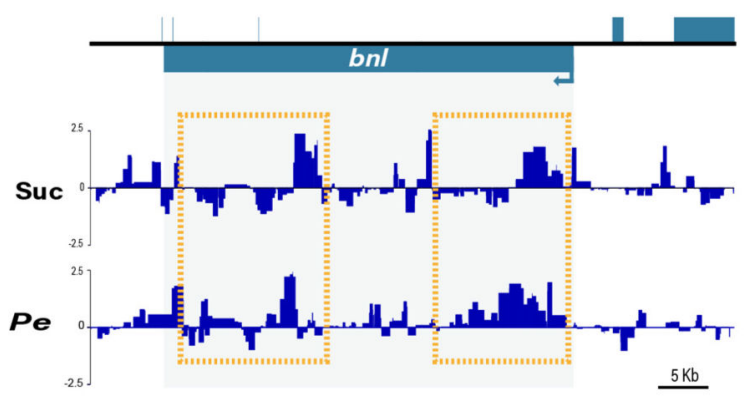

Extended Data Fig. 7. Genome wide RNA pol II binding profile within adult TTC by targeted DamID (TaDa).

a, Scatterplots indicating correlation between TaDa replicates for each of the conditions used in this study. Significant correlation is observed between replicates of each condition. Each data point represents the average score for each gene ( $\log 2$ Dam-pol II/Dam-only). b, RNA Pol II binding profile to $b n l$ in TTCs from Sucrose and Pe treated midguts. Boxes highlight areas with most prominent differences in RNA Pol II binding.

\section{Supplementary Material}

Refer to Web version on PubMed Central for supplementary material.

\section{Acknowledgements}

J.P. and J.B.C. are funded by a Wellcome Trust and Royal Society Sir Henry Dale Fellowship (Grant Number 104103/Z/14/Z; J.B.C.) and a Wellcome Trust Institutional Strategic Support Fund (ISSF) - Excellence and Innovation Catalyst Award to J.B.C. J.P. was partly funded by a BBSRC - Flexible Talent Mobility Account (FTMA) Award (BB/R506576/1). Y.Y and A.B.M. are funded by CRUK core funding to the CRUK Beatson Institute (A17196). T.D.S and G.N.A. were funded by a Wellcome Trust Investigator grant (104567; T.D.S.) and a BBSRC grant (BB/P017924/1; T.D.S. and G.N.A.)

We are thankful to Marta Llimargas, Markus Affolter, Irene Miguel-Aliaga, Andrea Brand, Cedric Polesello, Alessandro Scopelliti, Pablo Wappner, Ilan Davis, Florence Besse, Hugo Stoker, Gines Morata and Fisun Hamaratoglu Dion for generously sharing reagents and fly lines. We thank the Kyoto, Vienna and Bloomington Drosophila Stock Centres and the Drosophila Studies Hybridoma Bank for fly stocks and antibodies. We thank Colin Nixon (Beatson CRUK histology service) for IHC of mouse intestinal samples, Elaine McKenzie for help and training on the use of the hypoxia chamber, Margaret Mullin for assistance with TEM and David Strachan, John Halpin and Robert Insall (Beatson CRUK) for support with image quantification. We thank David McGuinness and Julie Galbraith (Glasgow Polyomics) for sequencing samples for TaDa and Rhoda Stefanatos for advice with RTqPCRs. We thank Maté Naszai for help with the creation of custom ImageJ macros and Lynsey Carroll for providing mouse intestinal samples. We thank Jean-Philippe Parvy and multiple members of the Cordero lab for scientific discussion and advice on the project.

\section{Data availability}

TaDa sequencing data including all raw sequence files and processed files have been deposited in the Gene Expression Omnibus under accession number GSE163570.

Public databases used in this study include: FlyBase (https://flybase.org) and ftp:// ftp.flybase.net/genomes/Drosophila_melanogaster/. Any additional data supporting this study will be made available by the corresponding author upon reasonable request. 


\section{Code availability}

The custom Perl script used for TaDa analysis ${ }^{47}$ is available at https://github.com/ tonysouthall/Dam-RNA_POLII_analysis. The custom ImageJ macros used in this study are available at: https://github.com/emltwc/TracheaProject. Any additional information on codes is available from the corresponding author upon request.

\section{References}

1. Beumer J, Clevers H. Regulation and plasticity of intestinal stem cells during homeostasis and regeneration. Development. 2016; 143:3639-3649. [PubMed: 27802133]

2. McCarthy N, Kraiczy J, Shivdasani RA. Cellular and molecular architecture of the intestinal stem cell niche. Nat Cell Biol. 2020; 22:1033-1041. [PubMed: 32884148]

3. Hageman JH, et al. Intestinal Regeneration: Regulation by the Microenvironment. Dev Cell. 2020; 54:435-446. [PubMed: 32841594]

4. Ghabrial A, Luschnig S, Metzstein MM, Krasnow MA. Branching morphogenesis of the Drosophila tracheal system. Annu Rev Cell Dev Biol. 2003; 19:623-647. [PubMed: 14570584]

5. Hayashi S, Kondo T. Development and Function of the Drosophila Tracheal System. Genetics. 2018; 209:367-380. [PubMed: 29844090]

6. Eilken HM, Adams RH. Dynamics of endothelial cell behavior in sprouting angiogenesis. Curr Opin Cell Biol. 2010; 22:617-625. [PubMed: 20817428]

7. Affolter M, et al. Tube or not tube: remodeling epithelial tissues by branching morphogenesis. Dev Cell. 2003; 4:11-18. [PubMed: 12530959]

8. Cela C, Llimargas M. Egfr is essential for maintaining epithelial integrity during tracheal remodelling in Drosophila. Development. 2006; 133:3115-3125. [PubMed: 16831830]

9. Centanin L, Gorr TA, Wappner P. Tracheal remodelling in response to hypoxia. J Insect Physiol. 2010; 56:447-454. [PubMed: 19482033]

10. Linneweber GA, et al. Neuronal control of metabolism through nutrient-dependent modulation of tracheal branching. Cell. 2014; 156:69-83. [PubMed: 24439370]

11. Casali A, Batlle E. Intestinal stem cells in mammals and Drosophila. Cell Stem Cell. 2009; 4:124127. [PubMed: 19200801]

12. Micchelli CA, Perrimon N. Evidence that stem cells reside in the adult Drosophila midgut epithelium. Nature. 2006; 439:475-479. [PubMed: 16340959]

13. Ohlstein B, Spradling A. The adult Drosophila posterior midgut is maintained by pluripotent stem cells. Nature. 2006; 439:470-474. [PubMed: 16340960]

14. Miguel-Aliaga I, Jasper H, Lemaitre B. Anatomy and Physiology of the Digestive Tract of Drosophila melanogaster. Genetics. 2018; 210:357-396. [PubMed: 30287514]

15. Li W, et al. MicroRNA-276a functions in ellipsoid body and mushroom body neurons for naive and conditioned olfactory avoidance in Drosophila. J Neurosci. 2013; 33:5821-5833. [PubMed: 23536094]

16. Sutherland D, Samakovlis C, Krasnow MA. branchless encodes a Drosophila FGF homolog that controls tracheal cell migration and the pattern of branching. Cell. 1996; 87:1091-1101. [PubMed: 8978613]

17. Guillemin K, et al. The pruned gene encodes the Drosophila serum response factor and regulates cytoplasmic outgrowth during terminal branching of the tracheal system. Development. 1996; 122:1353-1362. [PubMed: 8625824]

18. Gervais L, Casanova J. The Drosophila homologue of SRF acts as a boosting mechanism to sustain FGF-induced terminal branching in the tracheal system. Development. 2011; 138:1269-1274. [PubMed: 21385762]

19. Centanin L, et al. Cell autonomy of HIF effects in Drosophila: tracheal cells sense hypoxia and induce terminal branch sprouting. Dev Cell. 2008; 14:547-558. [PubMed: 18410730] 
20. Jiang H, et al. Cytokine/Jak/Stat signaling mediates regeneration and homeostasis in the Drosophila midgut. Cell. 2009; 137:1343-1355. [PubMed: 19563763]

21. Buchon N, Broderick NA, Poidevin M, Pradervand S, Lemaitre B. Drosophila intestinal response to bacterial infection: activation of host defense and stem cell proliferation. Cell Host Microbe. 2009; 5:200-211. [PubMed: 19218090]

22. Buchon N, Broderick NA, Chakrabarti S, Lemaitre B. Invasive and indigenous microbiota impact intestinal stem cell activity through multiple pathways in Drosophila. Genes Dev. 2009; 23:23332344. [PubMed: 19797770]

23. Apidianakis Y, Pitsouli C, Perrimon N, Rahme L. Synergy between bacterial infection and genetic predisposition in intestinal dysplasia. Proc Natl Acad Sci U S A. 2009; 106:20883-20888. [PubMed: 19934041]

24. Morel F, Renoux M, Lachaume P, Alziari S. Bleomycin-induced double-strand breaks in mitochondrial DNA of Drosophila cells are repaired. Mutat Res. 2008; 637:111-117. [PubMed: 17825327]

25. Amcheslavsky A, Jiang J, Ip YT. Tissue damage-induced intestinal stem cell division in Drosophila. Cell Stem Cell. 2009; 4:49-61. [PubMed: 19128792]

26. Tian A, Wang B, Jiang J. Injury-stimulated and self-restrained BMP signaling dynamically regulates stem cell pool size during Drosophila midgut regeneration. Proc Natl Acad Sci U S A. 2017; 114:E2699-E2708. [PubMed: 28289209]

27. Ren F, et al. Hippo signaling regulates Drosophila intestine stem cell proliferation through multiple pathways. Proc Natl Acad Sci U S A. 2010; 107:21064-21069. [PubMed: 21078993]

28. Kim CK, Yang VW, Bialkowska AB. The Role of Intestinal Stem Cells in Epithelial Regeneration Following Radiation-Induced Gut Injury. Curr Stem Cell Rep. 2017; 3:320-332. [PubMed: 29497599]

29. Chatterjee M, Ip YT. Pathogenic stimulation of intestinal stem cell response in Drosophila. J Cell Physiol. 2009; 220:664-671. [PubMed: 19452446]

30. Lavista-Llanos S, et al. Control of the hypoxic response in Drosophila melanogaster by the basic helix-loop-helix PAS protein similar. Mol Cell Biol. 2002; 22:6842-6853. [PubMed: 12215541]

31. Li J, Hobman TC, Simmonds AJ. Gawky (GW) is the Drosophila melanogaster GW182 homologue. Adv Exp Med Biol. 2013; 768:127-145. [PubMed: 23224968]

32. Cordero JB, Stefanatos RK, Scopelliti A, Vidal M, Sansom OJ. Inducible progenitor-derived Wingless regulates adult midgut regeneration in Drosophila. EMBO J. 2012; 31:3901-3917. [PubMed: 22948071]

33. Ha EM, et al. An antioxidant system required for host protection against gut infection in Drosophila. Dev Cell. 2005; 8:125-132. [PubMed: 15621536]

34. Hochmuth CE, Biteau B, Bohmann D, Jasper H. Redox regulation by Keap1 and Nrf2 controls intestinal stem cell proliferation in Drosophila. Cell Stem Cell. 2011; 8:188-199. [PubMed: 21295275]

35. Rey S, Semenza GL. Hypoxia-inducible factor-1-dependent mechanisms of vascularization and vascular remodelling. Cardiovasc Res. 2010; 86:236-242. [PubMed: 20164116]

36. Bacon NC, et al. Regulation of the Drosophila bHLH-PAS protein Sima by hypoxia: functional evidence for homology with mammalian HIF-1 alpha. Biochem Biophys Res Commun. 1998; 249:811-816. [PubMed: 9731218]

37. Chandel NS, et al. Reactive oxygen species generated at mitochondrial complex III stabilize hypoxia-inducible factor-1alpha during hypoxia: a mechanism of O2 sensing. J Biol Chem. 2000; 275:25130-25138. [PubMed: 10833514]

38. Ohshiro T, Saigo K. Transcriptional regulation of breathless FGF receptor gene by binding of TRACHEALESS/dARNT heterodimers to three central midline elements in Drosophila developing trachea. Development. 1997; 124:3975-3986. [PubMed: 9374395]

39. Horowitz A, Simons M. Branching morphogenesis. Circ Res. 2008; 103:784-795. [PubMed: 18845818]

40. Du L, et al. Unique patterns of organization and migration of FGF-expressing cells during Drosophila morphogenesis. Dev Biol. 2017; 427:35-48. [PubMed: 28502613] 
41. Johansson J, et al. RAL GTPases Drive Intestinal Stem Cell Function and Regeneration through Internalization of WNT Signalosomes. Cell Stem Cell. 2019; 24:592-607. e597 [PubMed: 30853556]

42. Muha V, Muller HA. Functions and Mechanisms of Fibroblast Growth Factor (FGF) Signalling in Drosophila melanogaster. Int J Mol Sci. 2013; 14:5920-5937. [PubMed: 23493057]

43. Biteau B, Jasper H. EGF signaling regulates the proliferation of intestinal stem cells in Drosophila. Development. 2011; 138:1045-1055. [PubMed: 21307097]

44. Buchon N, Broderick NA, Kuraishi T, Lemaitre B. Drosophila EGFR pathway coordinates stem cell proliferation and gut remodeling following infection. BMC Biol. 2010; 8:152. [PubMed: 21176204]

45. Xu N, et al. EGFR, Wingless and JAK/STAT signaling cooperatively maintain Drosophila intestinal stem cells. Dev Biol. 2011; 354:31-43. [PubMed: 21440535]

46. Marshall OJ, Southall TD, Cheetham SW, Brand AH. Cell-type-specific profiling of protein-DNA interactions without cell isolation using targeted DamID with next-generation sequencing. Nat Protoc. 2016; 11:1586-1598. [PubMed: 27490632]

47. Southall TD, et al. Cell-type-specific profiling of gene expression and chromatin binding without cell isolation: assaying RNA Pol II occupancy in neural stem cells. Dev Cell. 2013; 26:101-112. [PubMed: 23792147]

48. Sandoval J, et al. RNAPol-ChIP: a novel application of chromatin immunoprecipitation to the analysis of real-time gene transcription. Nucleic Acids Res. 2004; 32:e88. [PubMed: 15247321]

49. Medioni C, Ramialison M, Ephrussi A, Besse F. Imp promotes axonal remodeling by regulating profilin mRNA during brain development. Curr Biol. 2014; 24:793-800. [PubMed: 24656828]

50. Samuels TJ, Jarvelin AI, Ish-Horowicz D, Davis I. Imp/IGF2BP levels modulate individual neural stem cell growth and division through myc mRNA stability. Elife. 2020; 9

51. Chung S, Chavez C, Andrew DJ. Trachealess (Trh) regulates all tracheal genes during Drosophila embryogenesis. Dev Biol. 2011; 360:160-172. [PubMed: 21963537]

52. Isaac DD, Andrew DJ. Tubulogenesis in Drosophila: a requirement for the trachealess gene product. Genes Dev. 1996; 10:103-117. [PubMed: 8557189]

53. Wilk R, Weizman I, Shilo BZ. trachealess encodes a bHLH-PAS protein that is an inducer of tracheal cell fates in Drosophila. Genes Dev. 1996; 10:93-102. [PubMed: 8557198]

54. Tamamouna, et al. Remodelling of oxygen-transporting tracheoles drives intestinal regeneration and tumourigenesis in Drosophila. Nature Cell Biology. 2021

55. Holloway EM, et al. Differentiation of Human Intestinal Organoids with Endogenous Vascular Endothelial Cells. Dev Cell. 2020; 54:516-528. e517 [PubMed: 32841595]

56. Pasquier J, et al. Angiocrine endothelium: from physiology to cancer. J Transl Med. 2020; 18:52. [PubMed: 32014047]

57. Dai N, et al. IGF2BP2/IMP2-Deficient mice resist obesity through enhanced translation of Ucp1 mRNA and Other mRNAs encoding mitochondrial proteins. Cell Metab. 2015; 21:609-621. [PubMed: 25863250]

58. Zhou S, Degan S, Potts EN, Foster WM, Sunday ME. NPAS3 is a trachealess homolog critical for lung development and homeostasis. Proc Natl Acad Sci U S A. 2009; 106:11691-11696. [PubMed: 19581591]

59. Nusse YM, et al. Parasitic helminths induce fetal-like reversion in the intestinal stem cell niche. Nature. 2018; 559:109-113. [PubMed: 29950724]

60. Poplawski GHD, et al. Injured adult neurons regress to an embryonic transcriptional growth state. Nature. 2020; 581:77-82. [PubMed: 32376949]

61. Yui S, et al. YAP/TAZ-Dependent Reprogramming of Colonic Epithelium Links ECM Remodeling to Tissue Regeneration. Cell Stem Cell. 2018; 22:35-49. e37 [PubMed: 29249464]

62. Marshall OJ, Brand AH. damidseq_pipeline: an automated pipeline for processing DamID sequencing datasets. Bioinformatics. 2015; 31:3371-3373. [PubMed: 26112292]

63. Yu G, Wang LG, Han Y, He QY. clusterProfiler: an R package for comparing biological themes among gene clusters. OMICS. 2012; 16:284-287. [PubMed: 22455463] 
a

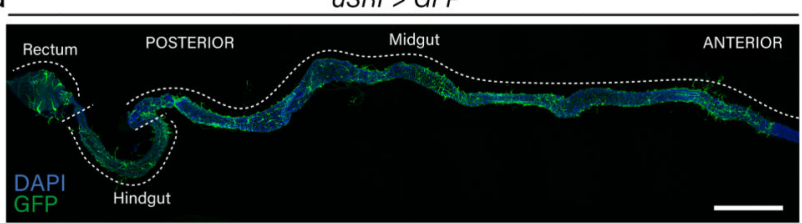

b

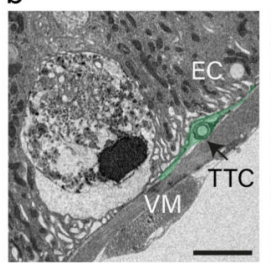

C

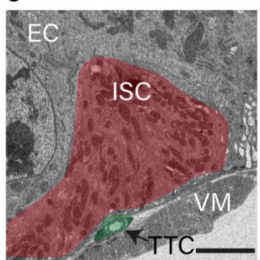

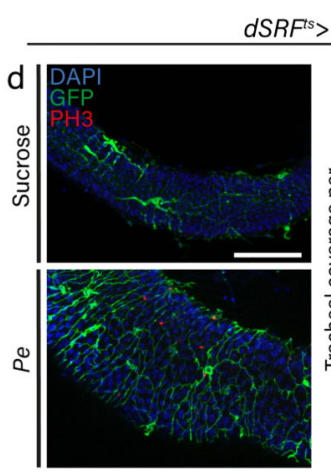

$S R F^{t s}>G F P$
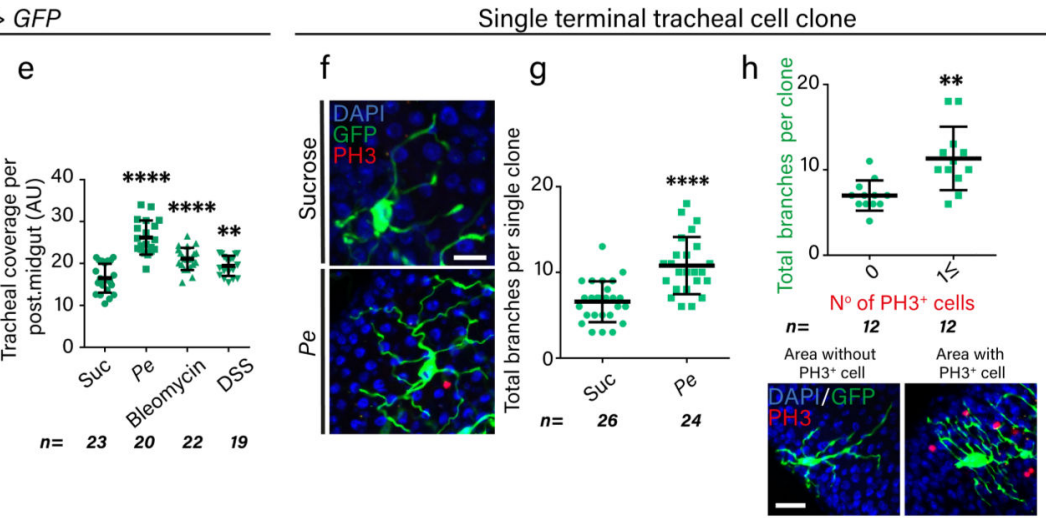

$d S R F^{t s}>$ GFP

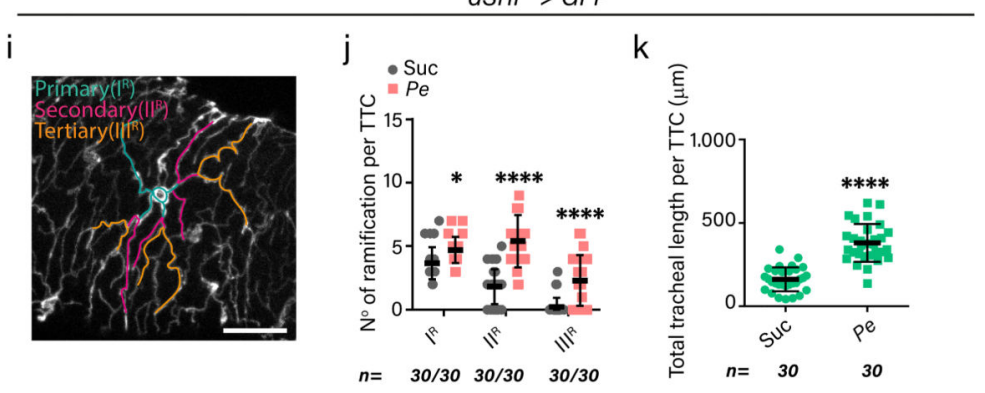

$\mathrm{m}$
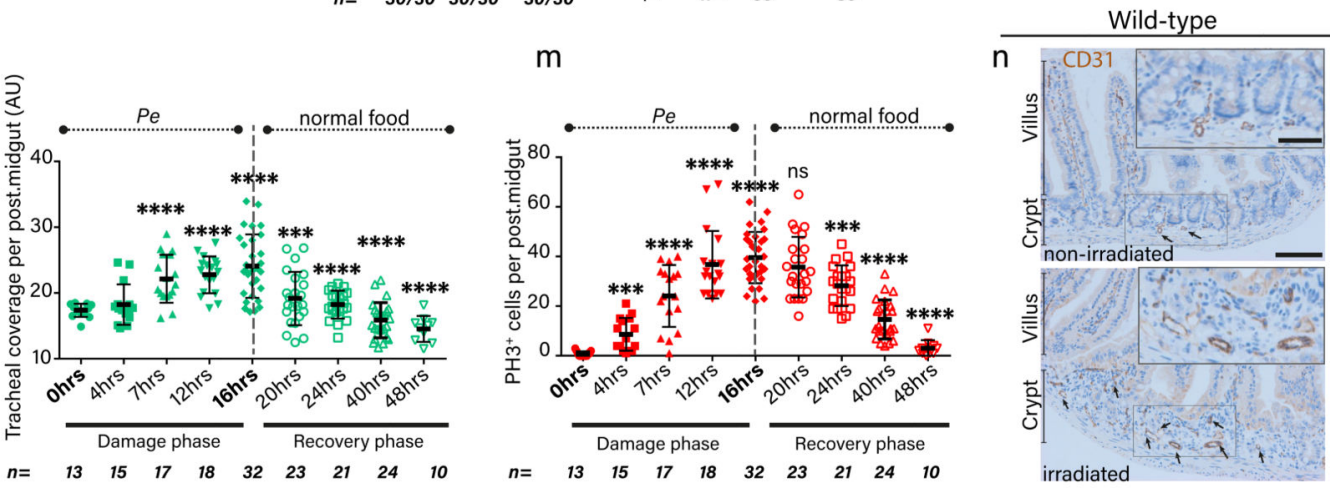

Fig. 1. Adult tracheal plasticity following intestinal injury.

a, Adult Drosophila gut-associated terminal tracheal cells (TTCs) (green). Scale bar: 500 $\mu \mathrm{m}$.

b, c, Transmission electron microscopy of adult posterior midguts: TTCs (green pseudocoloured), enterocyte (EC), intestinal stem cell (ISC) (red pseudo-coloured); VM: visceral muscle, Scale bars: $5 \mu \mathrm{m}$. d, TTCs (green) and proliferating ISCs (PH3; red) in control (Sucrose) or damaged $(P e)$ midguts. Scale bars: $100 \mu \mathrm{m}$. e, Tracheal coverage in midguts upon indicated treatments. Two-tailed, unpaired T-test $(* * * * P<0.0001, * * P=0.003)$; $\mathrm{n}=$ number of midguts, indicated in panel. f, Single TTC clones (green) and proliferating 
ISCs (PH3; red) from Sucrose or Pe midguts. Scale bar: $25 \mu \mathrm{m}$. g, Total number of branches per TTC clone in midguts as in (f). h, (top), Total number of branches per TTC clone and nearby $\mathrm{PH}^{+}$ISCs upon $\mathrm{Pe}$ infection. (bottom), TTC clones (green) and proliferating ISCs (PH3; red) used for quantifications in top panel. Scale bars: $25 \mu \mathrm{m} . \mathbf{g}, \mathbf{h}$ Two-tailed, unpaired T-test; $\mathrm{n}=$ number of TTC clones from 7-9 posterior midguts, indicated in panels. $\mathbf{g}$, $(* * * * P<0.0001) . \mathbf{h},(* * P=0.0014)$. i, TTC with pseudo-coloured primary $\left(\mathrm{I}^{\mathrm{R}}\right)$, secondary $\left(\mathrm{II}^{\mathrm{R}}\right)$ and tertiary $\left(\mathrm{III} \mathrm{R}^{\mathrm{R}}\right.$ ) branches. Scale bar: $50 \mu \mathrm{m}$. Image adapted from Fig. 1d (bottom panel). j, Number of branches per TTC from control or $P$ infected midguts. Two-way ANOVA and Sidak's multiple comparisons test $(* P<0.05, * * * * P<0.0001)$; $\mathrm{n}=$ number of TTCs from 6 midguts per condition, indicated in panel. $\mathbf{k}$, Total tracheal length per TTC. Two-tailed, unpaired T-test $(* * * * P<0.0001) ; \mathrm{n}=$ number of TTCs from 6 midguts per condition, indicated in panel. $\mathbf{l}, \mathbf{m}$, Tracheal coverage (l) and $\mathrm{PH} 3^{+}$cells (m) in midguts during 16 hrs of $P e$ infection followed by 32 hrs standard food consumption; $\mathrm{n}=$ number of midguts, indicated in panel. Two-tailed, unpaired T-test comparing each damage and recovery timepoint against the $0 \mathrm{hrs}$ and $16 \mathrm{hrs}$ time points, respectively. $\mathbf{l},(4 \mathrm{hrs} P=0.3446$, $7 \mathrm{hrs}, 12 \mathrm{hrs}, 16 \mathrm{hrs} * * * * P<0.0001,20 \mathrm{hrs} * * * P=0.0003,24 \mathrm{hrs}, 40 \mathrm{hrs}, 48 \mathrm{hrs} * * * * P<0.0001)$. $\mathbf{m},(4 \mathrm{hrs} * * * P=0.0002,7 \mathrm{hrs}, 12 \mathrm{hrs}, 16 \mathrm{hrs} * * * * P<0.0001,20 \mathrm{hrs} P=0.2055,24 \mathrm{hrs}$ $* * * P=0.0001,40 \mathrm{hrs}, 48 \mathrm{hrs} * * * * P<0.0001)$. $\mathbf{n}$, Endothelial cells (anti-CD31) in control (non-

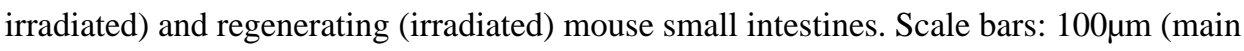
figure); $50 \mu \mathrm{m}$ (close up view).

Values represent mean \pm S.E.M. 

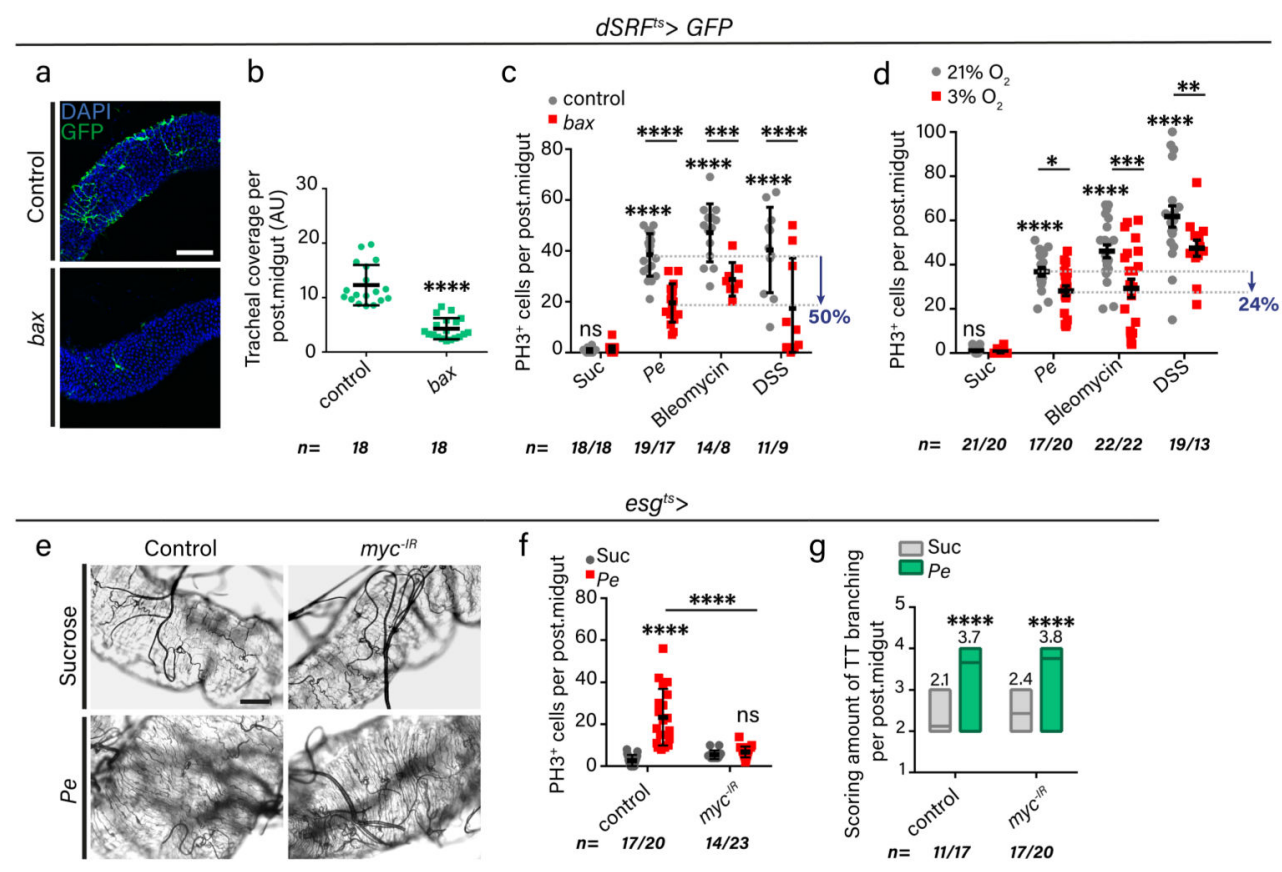

$e^{t s}>$
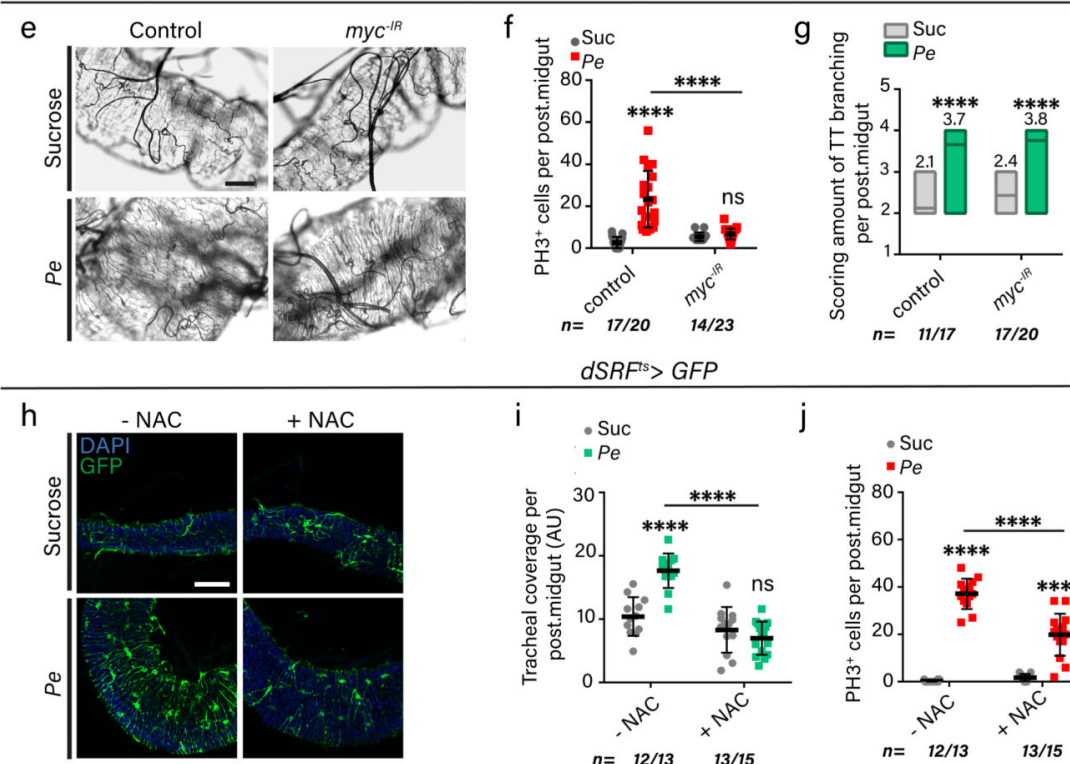

$d S R F^{t s}>$ GFP
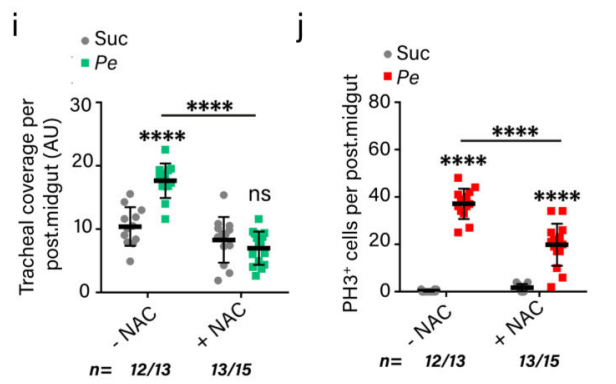

$d S R F>$ GFP; Idh-lacZ
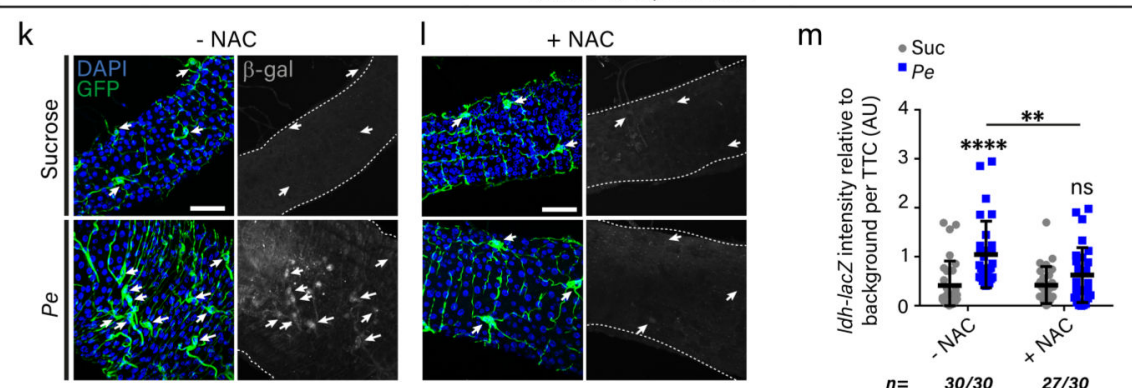

Fig. 2. ROS induced tracheal remodelling is required for intestinal regeneration.

a, Confocal images of adult posterior midguts from control animals or upon adult-specific bax-driven TTC loss (green). Scale bar: $100 \mu \mathrm{m}$. b, Quantification of tracheal coverage in midguts as in (a). Two-tailed, unpaired T-test $(* * * * P<0.0001) ; \mathrm{n}=$ number of midguts, indicated in panel. c-d, Quantification of $\mathrm{PH}^{+}$ISCs in posterior midguts of the genotypes and conditions indicated. Two-way ANOVA followed by Sidak's multiple comparisons test; $\mathrm{n}=$ number of midguts, indicated in panel. $\mathbf{c},(* * * * P<0.0001, * * * P<0.001) . \mathbf{d}$, $(* * * * P<0.0001, * * * P<0.001, * * P<0.01, * P<0.0267)$ e, Brightfield images of adult posterior 
midguts from Sucrose fed and $P e$ infected animals without (control) or with adult-specific overexpression of myc RNAi in ISCs/EBs (esgts $>$ ). Scale bar: 50 $\mu \mathrm{m} . \mathbf{f}, \mathbf{g}$, Quantification of $\mathrm{PH}^{+}$ISCs (f) and scored tracheal branching (g) in posterior midguts as in (e). Two-way ANOVA followed by Sidak's multiple comparisons test; $\mathrm{n}=$ number of midguts, indicated in panel. f, $(* * * * P<0.0001) . \mathbf{g},(* * * * P<0.0001) . \mathbf{h}$, Confocal images of Sucrose and $P e$ treated midguts in the presence or absence of the antioxidant NAC. Scale bar: $100 \mu \mathrm{m} . \mathbf{i}, \mathbf{j}$, Quantification of tracheal coverage (i) and $\mathrm{PH}^{+}$ISCs (j) in midguts as in (h). Two-way ANOVA followed by Sidak's multiple comparisons test; $n=$ number of midguts, indicated in panel. i, $(* * * * P<0.0001) . \mathbf{j},(* * * * P<0.0001)$. k,, , TTCs (green; white arrows) and Sima/ HIF-1a activity reporter $1 d h-l a c Z$ (grey; white arrows) in Sucrose fed or $P$ e infected adult posterior midguts without or with NAC (k, 1, respectively). Scale bar: 50 $\mu \mathrm{m}$. m, Quantification of the average $1 d h-l a c Z$ staining intensity in TTC within a defined region of posterior midguts as in $(\mathrm{k}, \mathrm{l})$. Two-way ANOVA followed by Sidak's multiple comparisons test $(* * * * P<0.0001, * * P<0.01) ; \mathrm{n}=$ number of TTC quantified from 9 to 10 posterior midguts per condition, indicated in panel.

Box plots represent maxima, minima and mean. Mean value is indicated on top of boxes. Otherwise, values represent mean \pm S.E.M. 


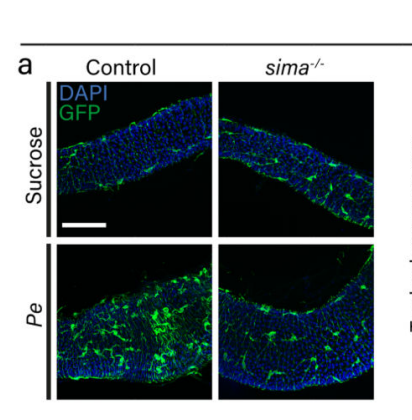

$d S R F>G F P$
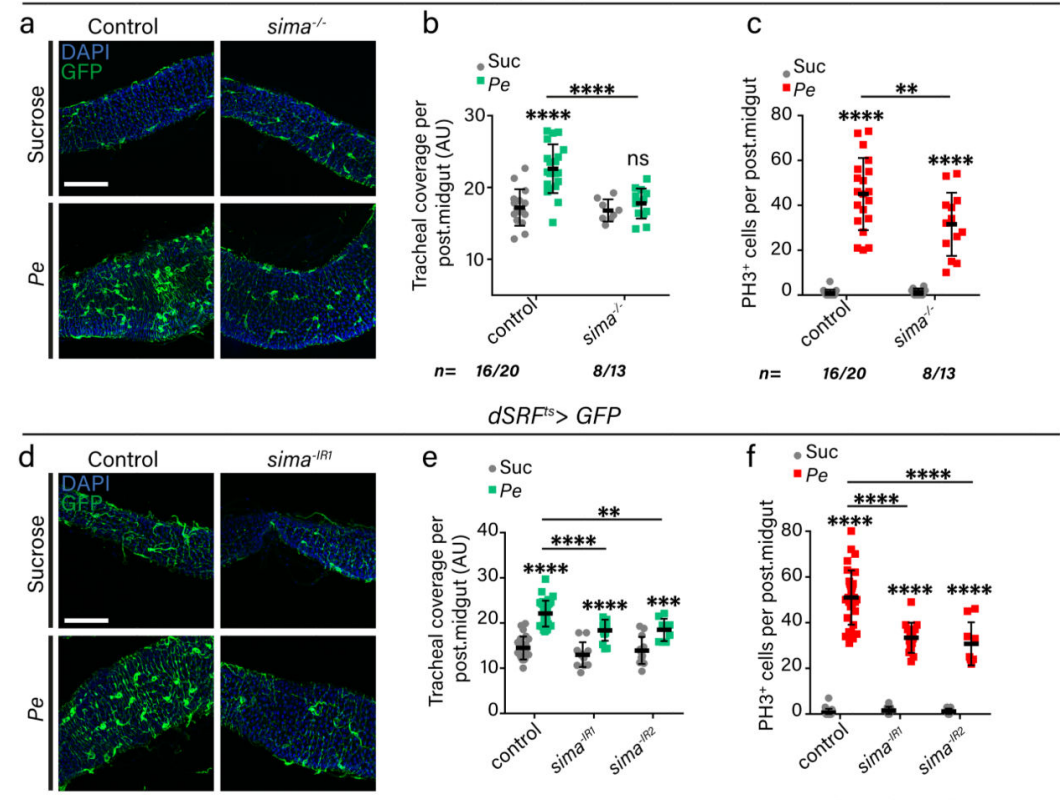

$d S R F^{s s}>$ GFP

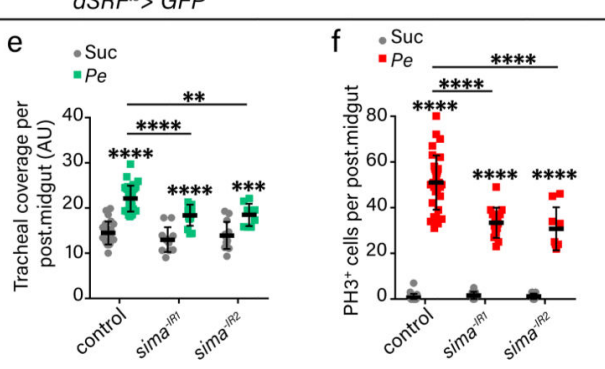

$n=24 / 26 \quad 12 / 15 \quad 13 / 9$

$n=24 / 26 \quad 12 / 15 \quad 13 / 9$
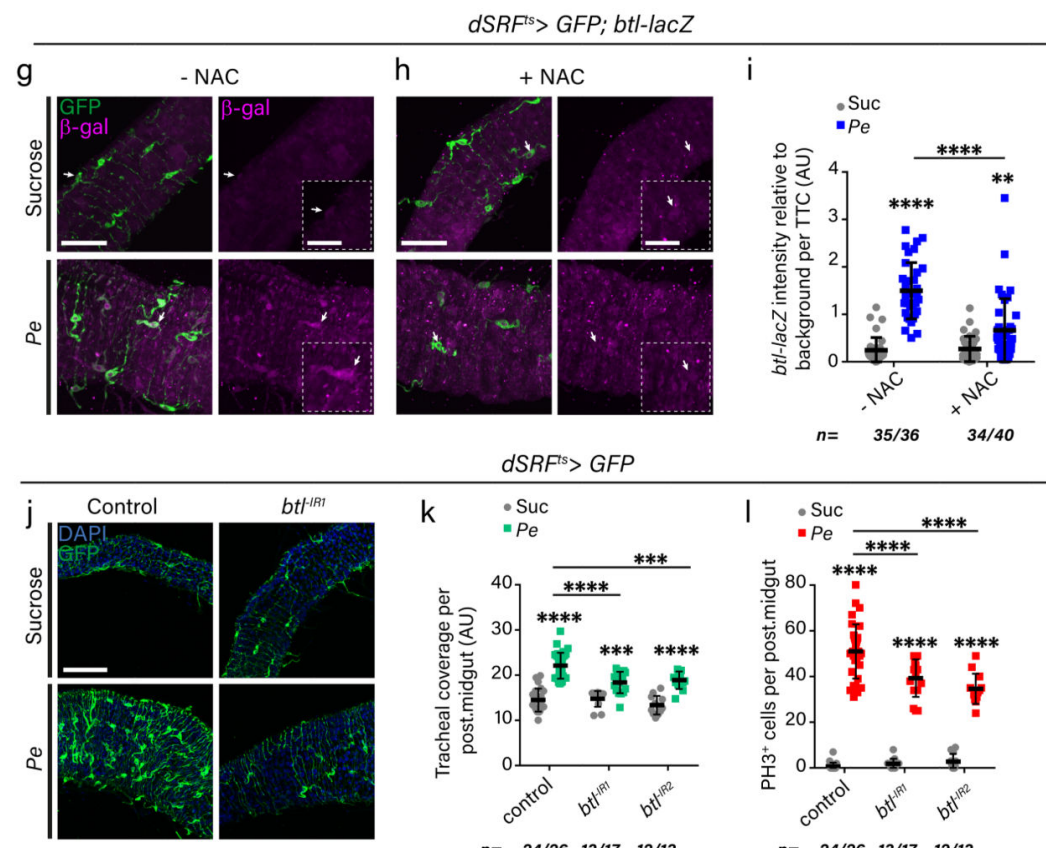

$d S R F^{i s}>G F P$

$n=35 / 36 \quad 34 / 40$
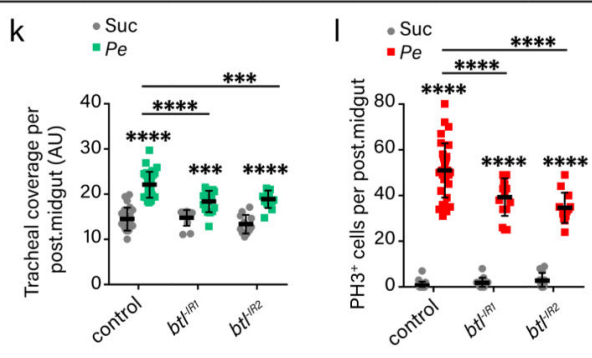

$n=\quad 24 / 26 \quad 13 / 17 \quad 12 / 13$

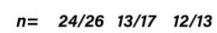

Fig. 3. Activation of HIF-1a/FGFR signaling in TTCs is required for tracheal remodelling and intestinal regeneration following damage.

a, Confocal images of TTCs (green) in control (Sucrose) or Pe infected adult posterior midguts from wild type or sima ${ }^{-/}$whole mutant animals. Scale bar: $100 \mu \mathrm{m}$. b, c,

Quantification of tracheal coverage (b) and $\mathrm{PH}^{+}$ISCs (c) in posterior midguts as in (a). d, Confocal images of Sucrose fed or Pe infected adult posterior midguts from wild type animals or animals (control) subject to sima RNAi overexpression $\left(\operatorname{sima}^{-I R}\right)$ within TTC $\left(d S R F^{t s}>G F P\right)$. Scale bar: $100 \mu \mathrm{m}$. e, f, Quantification of tracheal coverage (e) and $\mathrm{PH}^{+}$ 
ISCs in posterior midguts as in (d). a-f, Two-way ANOVA followed by Sidak's multiple comparisons test; $\mathrm{n}=$ number of midguts, indicated in panel. $\mathbf{b},(* * * * P<0.0001) . \mathbf{c}$, $(* * * * P<0.0001, * * \mathrm{p}<0.01) . \mathbf{e},(* * * * P<0.0001, * * * \mathrm{p}<0.001, * * \mathrm{p}<0.01) . \mathbf{f}$, $(* * * * P<0.0001)$. $\mathbf{g}, \mathbf{h}$, Confocal images of $b t$ l reporter expression (btl-lac $Z$; magenta) in Sucrose fed or Pe infected midguts without or with NAC (g, h, respectively). Dotted boxes represent a higher magnification of the area pointed with an arrow. Scale bars: 50 $\mu \mathrm{m}$ (main figures); $20 \mu \mathrm{m}$ (close up views). i, Quantification of btl-lac $Z$ staining intensity in TTC relative to background within a defined region of posterior midguts as in $(\mathrm{g}, \mathrm{h})$. Two-way ANOVA followed by Sidak's multiple comparisons test $(* * * * P<0.0001$, **p $<0.01)$; $\mathrm{n}=$ number of TTC from 9 to 10 posterior midguts/condition, indicated in panel. $\mathbf{j}$, Confocal images of Sucrose and $P$ e treated midguts from wild type animals (control) or following $R N A i$-driven $b t l$ knockdown $\left(b t t^{I R}\right)$ within TTCs. Scale bar: 100 $\mu \mathrm{m} . \mathbf{k}$, l, Quantification of tracheal coverage $(\mathrm{k})$ and $\mathrm{PH}^{+}{ }^{+} \mathrm{ISCs}$ in posterior midguts as in $(\mathrm{j})$. Two-way ANOVA followed by Sidak's multiple comparisons test; $\mathrm{n}=$ number of posterior midguts, indicated in panel. $\mathbf{k},(* * * * P<0.0001, * * * \mathrm{p}<0.001) . \mathbf{l},(* * * * P<0.0001)$.

Values represent mean \pm S.E.M. 


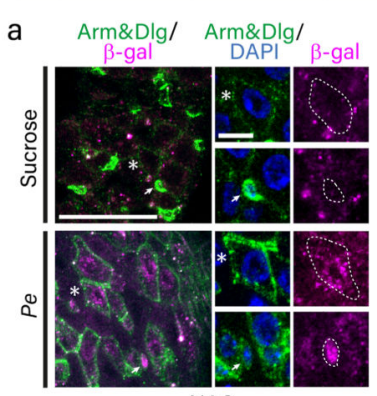

NAC
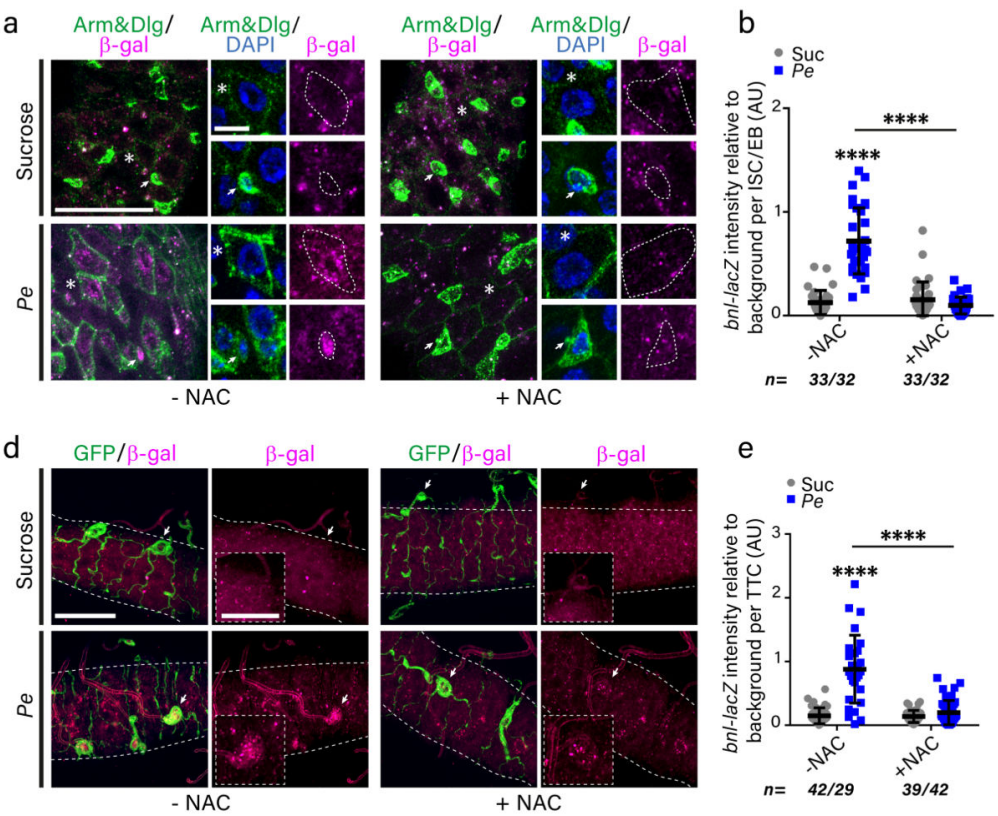

: Suc

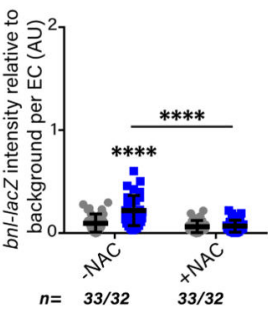

e

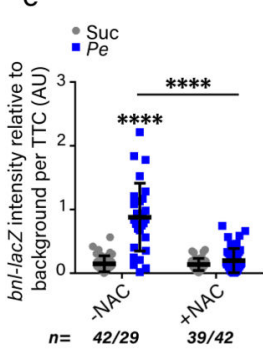

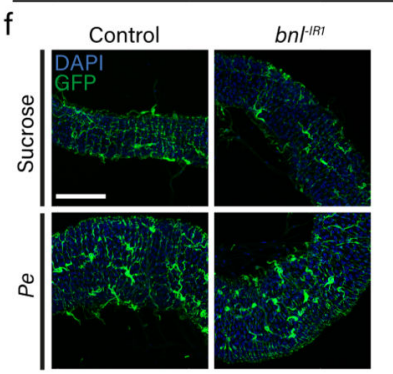

$d S R F^{t s}>$ GFP
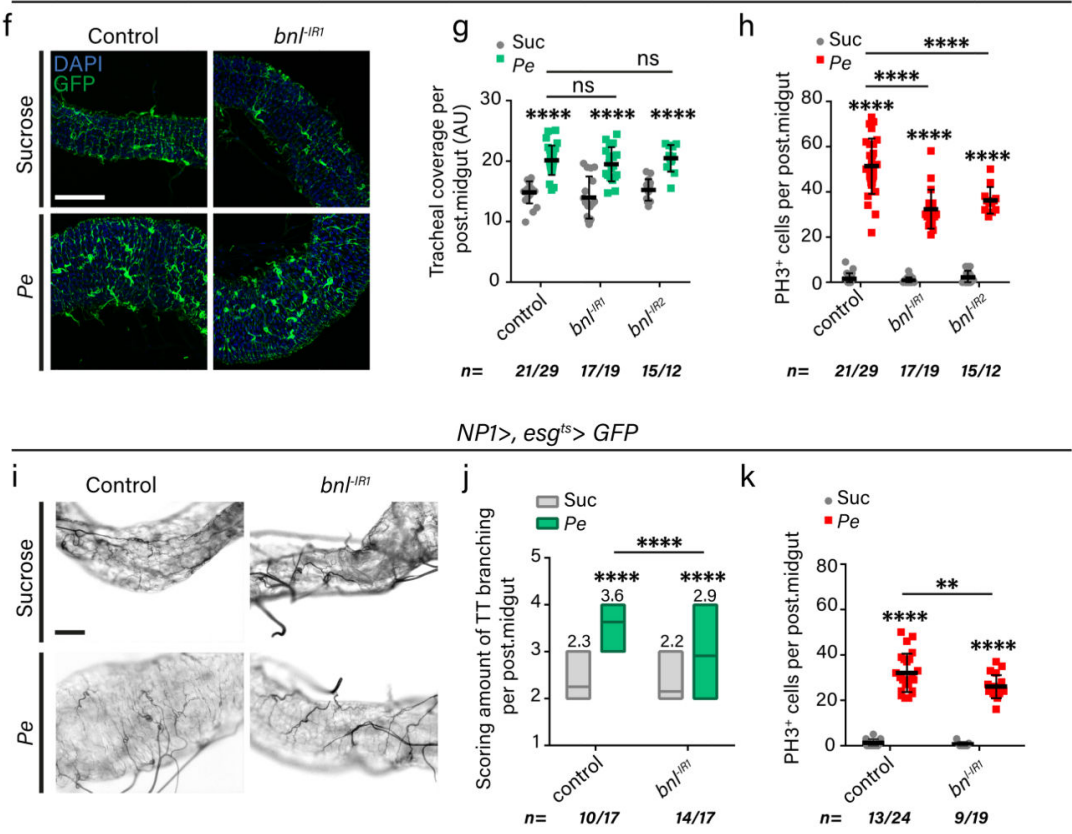

Fig. 4. Bidirectional FGF/FGFR signaling between the midgut and TTCs drives tracheal remodelling and ISC proliferation during intestinal regeneration.

a, $F G F / b n l$ reporter expression (bnl-lacZ; magenta) in Sucrose and $P e$ treated midguts in the presence or absence of NAC. Arrows point to reporter signal within ISCs/EBs (small nuclei and stained with anti-Armadillo (Arm); green pseudo-coloured). Asterisks point to reporter signal within ECs (large nuclei and stained with anti-Discs large (Dlg); green pseudocoloured). Dotted areas outline ISCs/EBs or ECs pointed by arrows or asterisks,

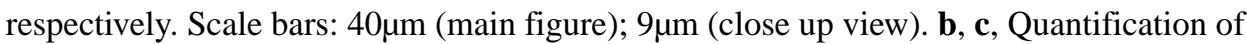


bnl-lacZ staining within ISCs/EBs (b) and ECs (c) in posterior midguts as in (a). d, Confocal images of $F G F / b n l$ reporter expression (bnl-lacZ; magenta) and TTCs ( $d S R F>G F P$, green) in Sucrose and $P e$ treated midguts in the absence or presence of NAC. Arrows indicate reporter signal within TTCs. Dotted boxes show a magnified view of TTCs pointed by arrows. Scale bars: $40 \mu \mathrm{m}$ (main figure); $20 \mu \mathrm{m}$ (close up view). e, Quantification of bnl-lac $Z$ staining within TTCs in posterior midguts as in (d). a-e, Two-way ANOVA followed by Sidak's multiple comparisons test; $\mathrm{n}=$ number of TTCs, ISC or ECs from 10-14 midguts per condition, indicated in panels. b, c, e, $(* * * * P<0.0001)$. f, Confocal images of Sucrose and $P e$ treated midguts from wild type animals (control) or upon $b n l$ knockdown $\left(b n r^{I R}\right)$ within TTCs. Scale bar: $50 \mu \mathrm{m} . \mathbf{g}, \mathbf{h}$, Quantification of tracheal coverage (g) and $\mathrm{PH}^{+}$ISCs (h) in midguts as in (f). Two-way ANOVA followed by Sidak's multiple comparisons test $(* * * * P<0.0001) ; \mathrm{n}=$ number of midguts, indicated in panels. $\mathbf{i}$, Brightfield images of Sucrose or $P e$ treated midguts from wild type animals (control) or upon $b n l$ knockdown $\left(b n r^{I R}\right)$ within ISCs/EBs and ECs $\left(N P 1>\right.$, esg $\left.g^{t s}>G F P\right)$. Scale bar: $50 \mu \mathrm{m} . \mathbf{j}, \mathbf{k}$, Quantification of scored tracheal branching (j) and $\mathrm{PH}^{+}$ISCs (k) in midguts as in (i). Two-way ANOVA and Sidak's multiple comparisons test; $\mathrm{n}=$ number of posterior midguts, indicated in the panels. j. $(* * * * P<0.0001) . \mathbf{k},(* * * * P<0.0001, * * \mathrm{p}<0.01)$.

Box plots represent maxima, minima and mean. Mean value is indicated on top of boxes. Otherwise, values represent mean \pm S.E.M. 
a

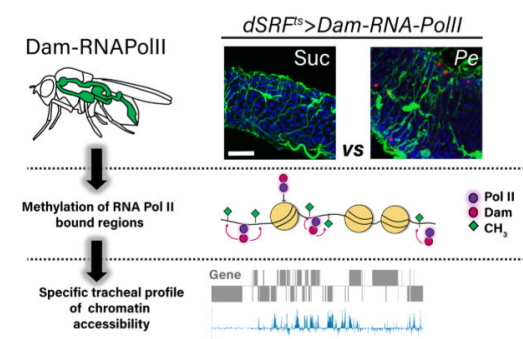

b

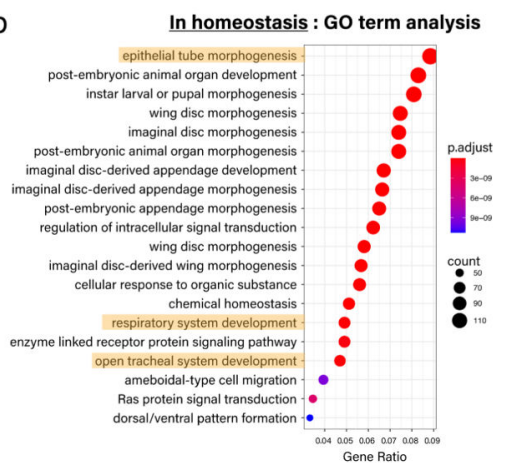

C

\begin{tabular}{|c|c|c|c|}
\hline & Gene & $\begin{array}{l}\text { Enrichment of } \\
\text { DamPol II/Dam } \\
\text { (Pe) (log2 ratio) }\end{array}$ & FDR \\
\hline Path & FBgn0036007 & 0.929 & 1.05E-05 \\
\hline Imp & FBgn0285926 & 0.462 & $1.26 \mathrm{E}-03$ \\
\hline ed & FBgn0000547 & 0.352 & $9.70 \mathrm{E}-14$ \\
\hline spen & FBgn0016977 & 0.344 & $2.30 \mathrm{E}-09$ \\
\hline Shep & FBgn0052423 & 0.277 & $3.80 \mathrm{E}-04$ \\
\hline
\end{tabular}

d

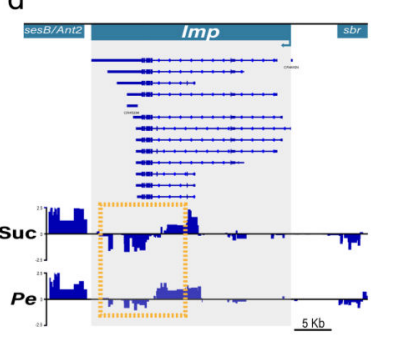

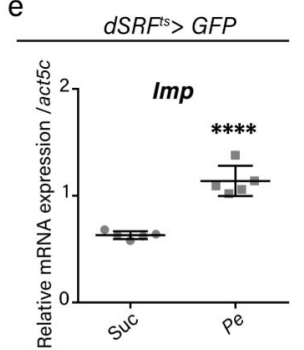

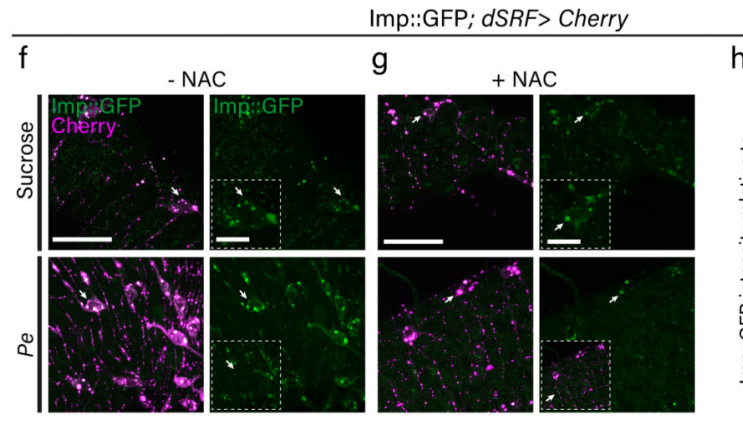

h

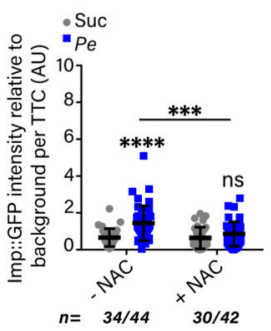

$d S R F^{1 s}>$ GFP
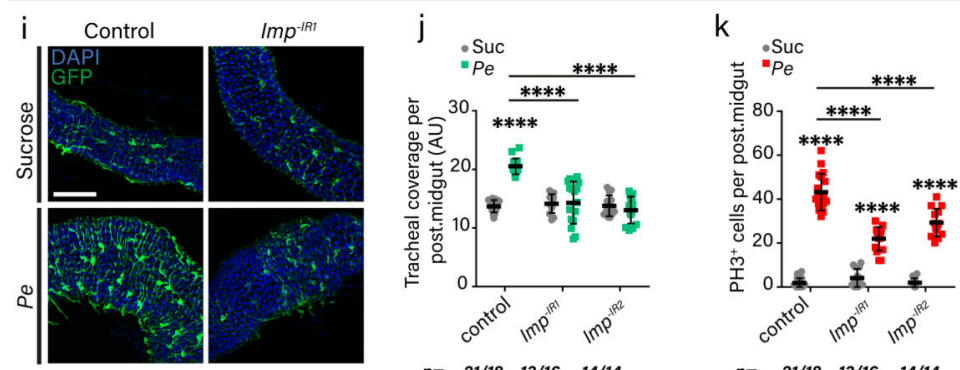

$n=21 / 18 \quad 13 / 16 \quad 14 / 14$

Fig. 5. Imp is a regulator of adult tracheal remodelling and intestinal regeneration.

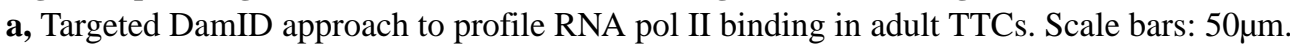
b, Gene Ontology term analysis corresponding to genes with significant RNA pol II binding in adult TTCs from control midguts (Sucrose). c, List of genes associated with neuronal processes showing significant RNA pol II binding in TTCs following Pe treatment. Data are presented as Log2 ratio of Dam-RNA Pol II/Dam reads; false discover rate (FDR). Monte Carlo simulations were performed using randomised data to generate FDR. d, Profile of RNA Pol II binding to Imp in TTCs from Sucrose (Suc) and Pe treated midguts. Boxes 
highlight areas with most prominent differences in RNA Pol II binding. e, RT-qPCR for Imp mRNA expression from whole adult midguts and associated tracheal tissue, in Sucrose or $P e$ fed animals. Two-tailed, unpaired T-test $(* * * * P<0.0001) ; \mathrm{n}=5$ biological replicates. f, $\mathbf{g}$, Confocal images of Imp protein reporter Imp::GFP (green; arrows) and TTCs

( $d S R F>C h e r r y$, magenta; arrows) in Sucrose and Pe treated midguts in the absence (f) or presence (g) of NAC. Dotted boxes show magnified views of TTCs pointed by arrows. Scale bars: $50 \mu \mathrm{m}$ (main figure); $12 \mu \mathrm{m}$ (close up view). h, Quantification of Imp::GFP staining intensity relative to background within TTCs in posterior midguts as in (f) and (g). Two-way ANOVA followed by Sidak's multiple comparisons test; $(* * * * P<0.0001$, $* * * \mathrm{p}<0.001)$; $\mathrm{n}=$ number of TTC from 11-14 posterior midguts per condition, indicated in panel. i, Sucrose or $P e$ treated midguts from wild type animals (control) or upon RNAi-driven adult-specific Imp

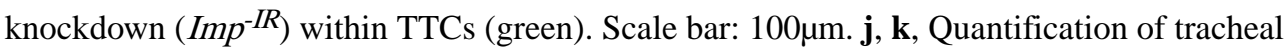
coverage (j) and $\mathrm{PH}^{+}{ }^{+}$ISCs (k) in posterior midguts as in (i). Two-way ANOVA and Sidak's multiple comparisons test $(* * * * P<0.0001) ; \mathrm{n}=$ number of posterior midguts, indicated in panels.

Values represent mean \pm S.E.M. 

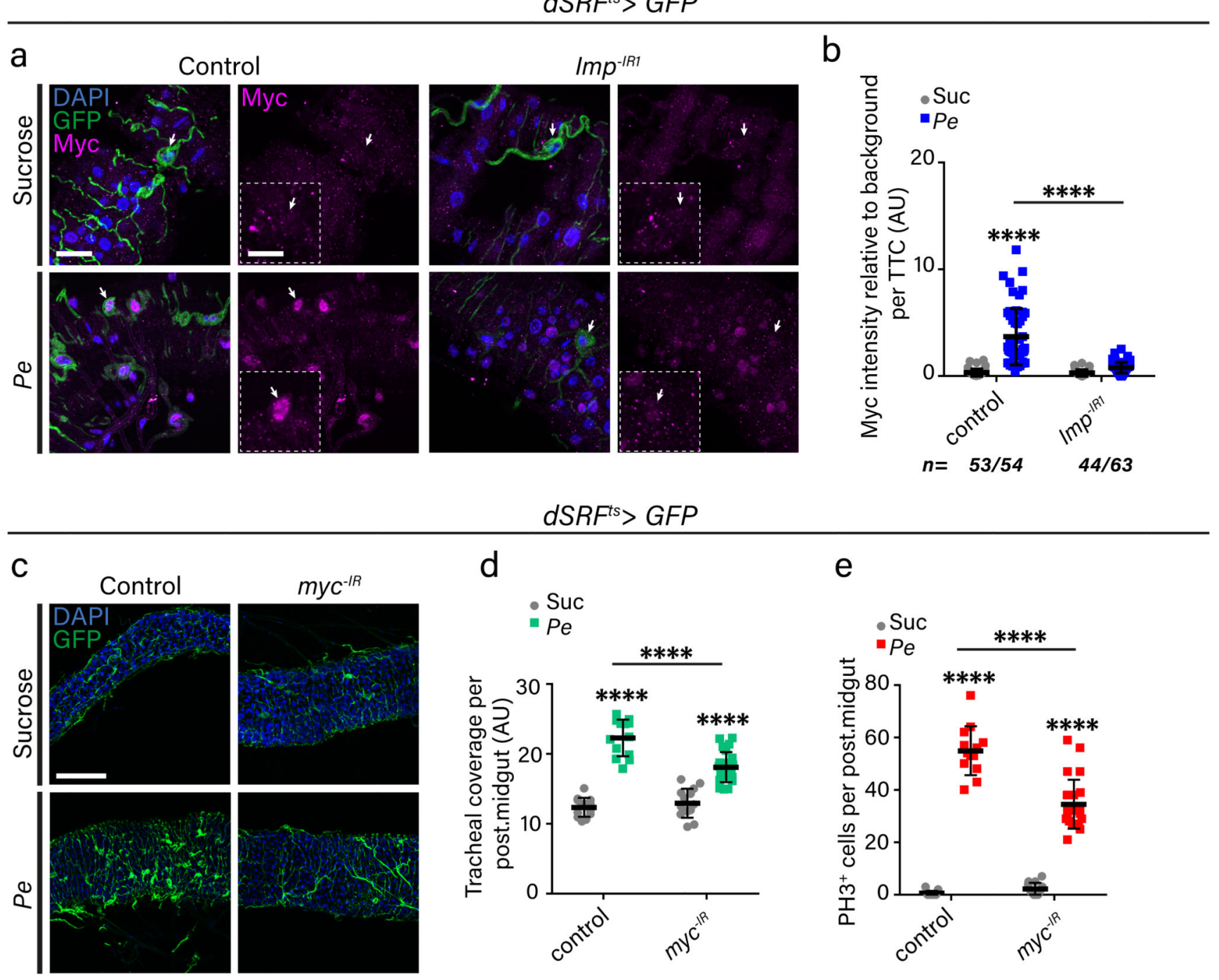

d

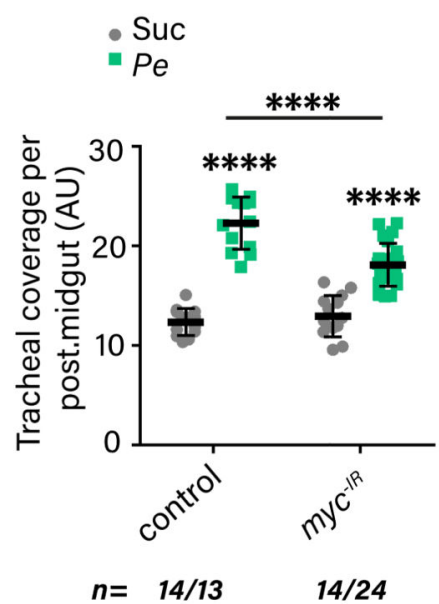

e

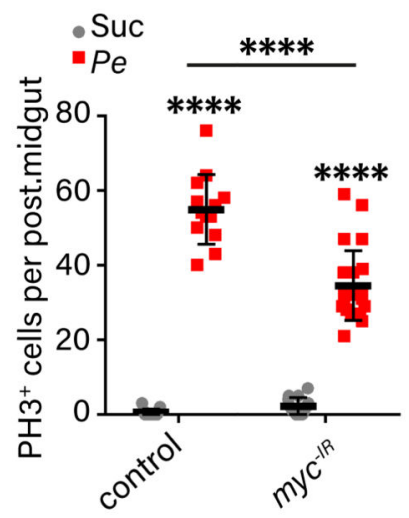

$n=14 / 13$

$14 / 24$

Fig. 6. Imp dependent activation of Myc in TTCs is required for tracheal remodelling and intestinal regeneration.

a, Confocal images of Myc staining (magenta; arrows) and TTCs ( $d S R F>G F P$, green; arrows) in Sucrose or Pe treated midguts from wild type animals (control) or upon RNAidriven adult-specific Imp knockdown $\left(I m p^{-I R}\right)$ within TTCs. Dotted boxes show magnified

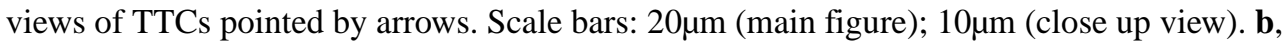
Quantification of Myc staining intensity relative to background in TTCs from posterior midguts as in (a). Two-way ANOVA followed by Sidak's multiple comparisons test $(* * * * P<0.0001) ; \mathrm{n}=$ number of TTCs from $15-18$ posterior midguts per condition, indicated in panel. c, Confocal images of Sucrose or Pe treated midguts from wild type animals (control) or upon RNAi-driven adult-specific $m y c$ knockdown $\left(m y c^{-I R}\right)$ within TTCs

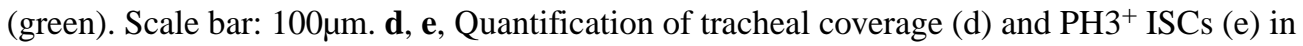
posterior midguts as in (c). Two-way ANOVA followed by Sidak's multiple comparisons test $(* * * * P<0.0001)$; $\mathrm{n}=$ number of midguts quantified, indicated in panels. 
Values represent mean \pm S.E.M. 
a

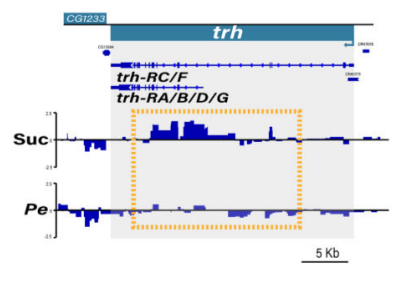

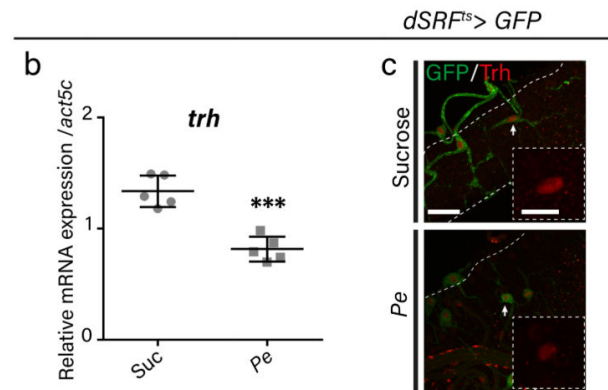

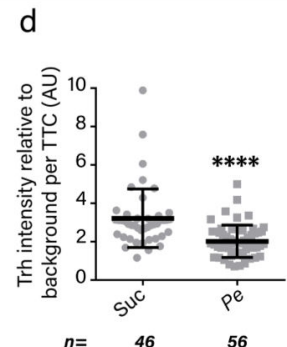

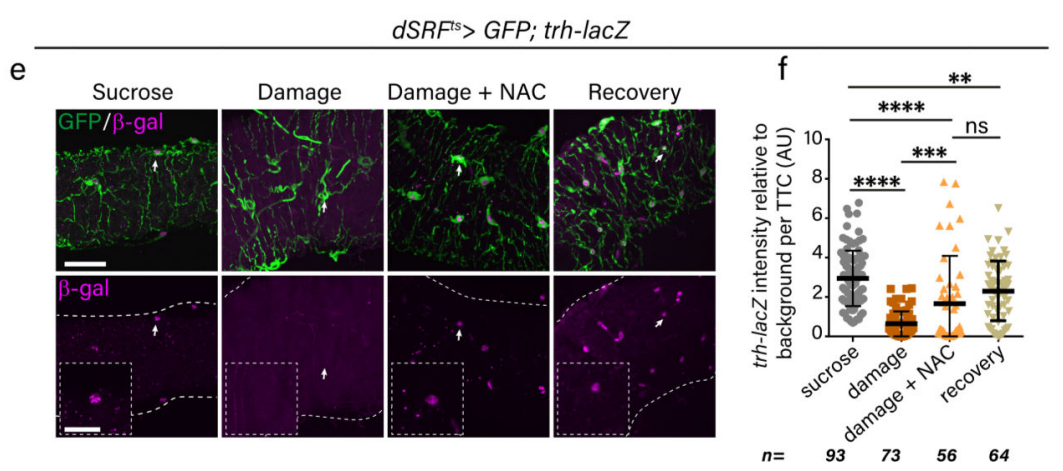

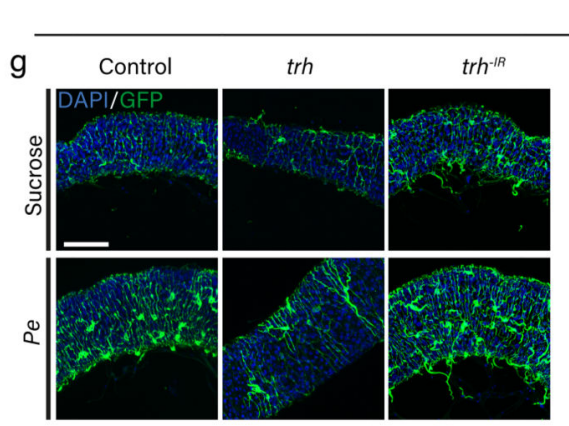

$d S R F^{t s}>$ GFP
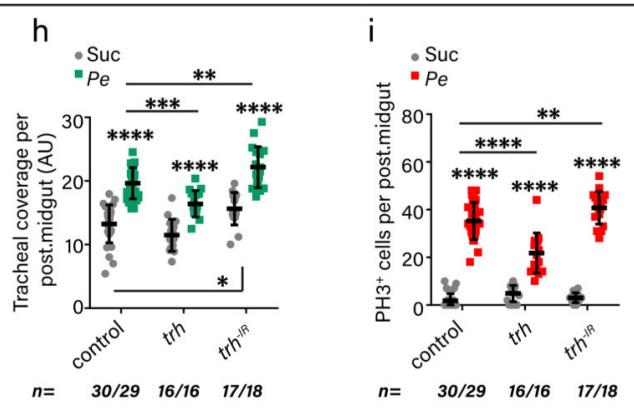

j DAMAGE intestinal epithelium

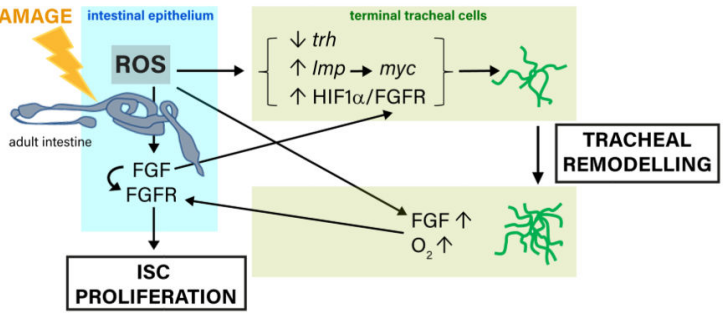

Fig. 7. Trachealess downregulation in TTCs is necessary for adult tracheal remodelling and damage induced ISC proliferation.

a, Profile of RNA Pol II binding to trachealess (trh) within TTCs of Sucrose (Suc) and Pe treated midguts. b, RT-qPCR for trh mRNA expression from whole adult midguts and associated tracheal tissue of Sucrose or Pe fed animals. Two-tailed, unpaired T-test (*** $P=0.0002) ; \mathrm{n}=5$ biological replicates. c, Confocal images of Trh staining (red; arrow) and TTCs ( $d S R F>G F P$, green; arrow) in Sucrose or Pe treated midguts. Dotted boxes show magnified views of TTCs pointed by arrows. Scale bars: $20 \mu \mathrm{m}$ (main figure); $10 \mu \mathrm{m}$ (close 
up view). d, Quantification of Trh staining intensity relative to background within TTCs in posterior midguts as in (c). Two-tailed, unpaired T-test $(* * * * P<0.0001)$; $\mathrm{n}=$ number of TTCs from 14 midguts per condition, indicated in panel. e, Confocal images of trh reporter expression (trh-lacZ; magenta; arrows) and TTCs ( $d S R F>G F P$, green; arrows) in Sucrose or $P e$ treated midguts without (Damage) or with NAC (Damage + NAC), and following normal food consumption during $32 \mathrm{hrs,} \mathrm{post-infection} \mathrm{(Recovery).} \mathrm{Dotted} \mathrm{boxes} \mathrm{show} \mathrm{magnified}$ views of TTCs pointed by arrows. Scale bars: $50 \mu \mathrm{m}$ (main figure); $20 \mu \mathrm{m}$ (close up view). f, Quantification of trh-lacZ staining intensity relative to background within TTCs of midguts as in (e). Two-tailed, unpaired T-test (damage $v s$ sucrose $* * * P<0.0001$, damage + NAC $v S$ damage $* * * P=0.0006$, recovery $v s$ damage + NAC $P=0.079$, damage + NAC $v s$ sucrose $* * * * P<0.0001$, recovery $v s$ sucrose $* * P=0.0066) ; \mathrm{n}=$ number of TTCs from 12-21 midguts per condition, indicated in panel. g, Confocal images from Sucrose or Pe treated midguts from wild type animals (control) or upon trh overexpression (trh) or RNAi-driven knockdown $\left(t r h^{-I R}\right)$ within TTCs (green). Scale bar: $100 \mu \mathrm{m} . \mathbf{h}, \mathbf{i}$, Quantification of tracheal coverage (h) and $\mathrm{PH}^{+}$ISCs (i) in midguts as in (g). Two-way ANOVA, followed by Sidak's multiple comparisons test $(* P<0.05, * * P<0.01, * * * P<0.001 * * * * P<0.0001) ; \mathrm{n}=$ number of posterior midguts, indicated in panels. $\mathbf{j}$, Schematic representation of working model. Values represent mean \pm S.E.M. 